\title{
Charge-orbital ordering and phase separation in the two-orbital model for manganites: Roles of Jahn-Teller phononic and Coulombic interactions
}

\author{
Takashi Hotta \\ Institute for Solid State Physics, University of Tokyo, 5-1-5 Kashiwa-no-ha, Kashiwa, Chiba 277-8581, Japan \\ Andre Luiz Malvezzi \\ Departamento de Física, Faculdade de Ciências, Universidade Estadual Paulista, Caixa Postal 473, 17.033-360, Bauru, SP, Brazil \\ Elbio Dagotto \\ Department of Physics and National High Magnetic Field Laboratory, Florida State University, Tallahassee, Florida 32306
}

(Received 23 February 2000)

\begin{abstract}
The main properties of realistic models for manganites are studied using analytic mean-field approximations and computational numerical methods, focusing on the two-orbital model with electrons interacting through Jahn-Teller (JT) phonons and/or Coulombic repulsions. Analyzing the model including both interactions by the combination of the mean-field approximation and the exact diagonalization method, it is argued that the spin-charge-orbital structure in the insulating phase of the purely JT-phononic model with a large Hund coupling $J_{\mathrm{H}}$ is not qualitatively changed by the inclusion of the Coulomb interactions. As an important application of the present mean-field approximation, the CE-type antiferromagnetic state, the charge-stacked structure along the $z$ axis, and $\left(3 x^{2}-r^{2}\right) /\left(3 y^{2}-r^{2}\right)$-type orbital ordering are successfully reproduced based on the JT-phononic model with large $J_{\mathrm{H}}$ for the half-doped manganite, in agreement with recent Monte Carlo simulation results. Topological arguments and the relevance of the Heisenberg exchange among localized $t_{2 \mathrm{~g}}$ spins explains why the inclusion of the nearest-neighbor Coulomb interaction does not destroy the charge stacking structure. It is also verified that the phase-separation tendency is observed both in purely JT-phononic (large $J_{\mathrm{H}}$ ) and purely Coulombic models in the vicinity of the hole undoped region, as long as realistic hopping matrices are used. This highlights the qualitative similarities of both approaches and the relevance of mixedphase tendencies in the context of manganites. In addition, the rich and complex phase diagram of the two-orbital Coulombic model in one dimension is presented. Our results provide robust evidence that Coulombic and JT-phononic approaches to manganites are not qualitatively different ways to carry out theoretical calculations, but they share a variety of common features.
\end{abstract}

\section{INTRODUCTION}

The recent discovery of the colossal magnetoresistance (CMR) phenomenon in manganites has triggered a huge experimental and theoretical effort to understand its origin. ${ }^{1,2}$ The large changes in resistivity observed in experiments usually involve both a low-temperature or high magnetic-field ferromagnetic (FM) metallic phase, and a high-temperature or low magnetic-field insulating phase. For this reason, to address the CMR effect, it appears unavoidable to have a proper understanding of the phases competing with the metallic FM regime, which as a first approximation can be rationalized based on the standard double-exchange mechanism. ${ }^{3,4}$ In fact, the phase diagram of manganites has revealed a very complex structure, clearly showing that metallic ferromagnetism is just one of the several spin, orbital, and charge arrangements that are possible in manganites. 5,6

Theoretical study of manganites started decades ago when the double-exchange ideas to explain the FM phase were proposed. Unfortunately, the model used in those early calculations involved only one orbital, and the many-body techniques used in its analysis were mostly restricted to crude mean-field approximations (MFA). Quite recently, computational studies of the one-orbital model for manganites have been presented by Yunoki et al. ${ }^{7-9}$ While the expected FM phase emerged clearly from such analysis, several features were identified, notably phase-separation (PS) tendencies close to $n=1$ (where $n$ is the $e_{\mathrm{g}}$ electron number density per site) between an antiferromagnetic (AFM) insulating phase and a metallic FM state. Several calculations have confirmed these tendencies toward mixed-phase characteristics, using a variety of techniques. ${ }^{10}$ Moreover, the computational work has been extended to include noncooperative Jahn-Teller (JT) phonons ${ }^{11}$ and PS tendencies involving spin-FM phases that differ in their orbital arrangement, i.e., staggered vs uniform, were again identified using one-dimensional (1D) and two-dimensional (2D) clusters. This illustrates the relevance of the orbital degree of freedom in the analysis of manganites, and confirms the importance of mixed-phase characteristics in this context. The theoretical, mostly computational, calculations have been recently summarized by Moreo et $a l .,{ }^{12}$ where it has been argued that a state with clusters of one phase embedded into another should have a large resistivity and a large compressibility. Other predictions in mixed-phase regimes involve the presence of a robust pseudogap in the density of states, ${ }^{13}$ similar to the results obtained using angle-resolved photoemission experiments applied to bilayer manganites. ${ }^{14}$ A large number of experiments ${ }^{12}$ have reported the presence of inhomogeneities in real manganites, ${ }^{15-17}$ results compatible with those obtained using computational studies. More recently, the influence of disorder on metal-insulator transitions of manganite models that would be of first-order character without disor- 
der has been proposed ${ }^{18}$ as the origin of the large cluster coexistence reported in experiments for several compounds. ${ }^{16,17}$ It is clear that intrinsic tendencies toward inhomogeneous states is at the heart of manganite physics, and simple scenarios involving small polarons are not sufficient to understand the mixed-phase tendencies observed experimentally.

Although the presence of mixed-phase tendencies competing with the FM phase is by now well established and a considerable progress has been achieved in the theoretical study of models for manganites, many issues still remain to be investigated in this context. One of them is related with the notorious charge-ordering phenomenon, characteristic of the narrow-band manganites. Especially at $n=0.5$, the socalled CE-type AFM phase has been widely observed in materials such as $\mathrm{La}_{0.5} \mathrm{Ca}_{0.5} \mathrm{MnO}_{3}$ and $\mathrm{Nd}_{0.5} \mathrm{Sr}_{0.5} \mathrm{MnO}_{3}$. In spite of the considerable importance of this phase in view of its relevance for the CMR effect reported at high hole density, ${ }^{2}$ it is only recently that the $\mathrm{CE}$ phase has been theoretically understood using the analytic MFA in the JT-phononic model $^{19,20}$ and numerical computational techniques. ${ }^{21,22}$ The importance of the zigzag chains of the CE state, along which the spins are parallel, has been remarked in these recent efforts. In fact, it has been shown that even without electronphonon or Coulomb interactions the CE state structure is nevertheless stable, and in this limit it arises from a simple band-insulator picture. Previous work, such as the pioneering results of Goodenough, ${ }^{23}$ were based on the assumption of a checkerboard charge state upon which the spin and orbital arrangements were properly calculated, but they did not consider the competition with other charge-disordered phases in a fully unbiased calculation as carried out in Refs. 19-22.

Here a particular feature of the charge-ordered (CO) CEphase structure should be noted. In the $x-y$ plane, the $\mathrm{Mn}^{3+}$ and $\mathrm{Mn}^{4+}$ ions are arranged in a checkerboard pattern, which naively seems to arise quite naturally from the presence of a strong long-range Coulomb interaction. ${ }^{23}$ However, contrary to this naive expectation, the same $\mathrm{CO}$ pattern stacks in experiments along the $z$ axis without any change, although the $t_{2 \mathrm{~g}}$ spin direction alternates from plane to plane. In other words, the $\mathrm{Mn}^{3+}$ and $\mathrm{Mn}^{4+}$ ions in the CE-type AFM structure do not form a three-dimensional (3D) Wigner-crystal structure, clearly suggesting that the origin of the $\mathrm{CO}$ phase in the half-doped manganite cannot be caused exclusively by a dominant long-range Coulomb interaction. Other interactions should be important as well. In this work, it is shown that the charge-stacked $\mathrm{CO}$ state in the CE-type structure found in experiments can also be obtained in the simple MFA for the purely JT-phononic model with a large Hund coupling $J_{\mathrm{H}}$. In addition, it is shown that this structure is not easily destroyed by the inclusion of the nearest-neighbor Coulomb interaction. The exchange $J_{\mathrm{AF}}$ between the $t_{2 \mathrm{~g}}$ spins plays a key role in the stabilization of the chargestacked structure. The details of the calculation are discussed here. The present semianalytical results are in excellent agreement with recent Monte Carlo simulations ${ }^{22}$ and provide a simple formalism to rationalize these computational results.

On the other hand, it is possible to take another approach to understand the $\mathrm{CO}$ state in manganites including the $\mathrm{CE}$ type structure, by emphasizing the role of the on-site Cou- lomb interaction. ${ }^{24}$ In this direction of study, several results are available in the literature. For instance, Ishihara et al. discussed the spin-charge-orbital ordering from the limit of the infinitely strong electronic correlation, namely, under the approximation of no double occupancy. ${ }^{25}$ Maezono et al. provided information on the overall features of the phase diagram for manganites by using a mean-field calculation. ${ }^{26}$ These two mean-field approaches do not reproduce the CE state of half-doped manganites. Mizokawa and Fujimori discussed the stabilization of the CE-type structure by using the model Hartree-Fock approximation. ${ }^{27}$ However, to the best of our knowledge, the origin of the charge-stacked structure in the CE-type state has not been clarified based on the Coulombic scenario, although several properties of manganites have been reproduced. In this paper, a possible way to understand the CE-type state with the charge-stacked structure in the purely Coulombic model, even without the long-range repulsion, is briefly discussed.

The present paper contains information about other subjects as well. In spite of the importance of additional interactions besides the long-range Coulombic one to stabilize the proper charge-stacked $n=0.5$ state, as discussed before, it is clear that at least the on-site Coulomb interactions are very strong and their influence should be considered in realistic calculations. Most of the previous analytic and computational works that reported the PS tendency and the $\mathrm{CO}$ phase have used electrons interacting among themselves indirectly through their coupling with localized $t_{2 \mathrm{~g}}$ spins or with JT phonons. The JT-phononic model, supplemented by a large Hund coupling, has been extensively studied by the present authors since the explicit inclusion of on-site Coulomb interactions diminish dramatically the feasibility of the computational work. In addition, it has been found that the JTphononic model explains quite well several experimental results, even if the Coulomb interaction is not included explicitly. Moreover, it should be noted that the energy gain due to the static JT distortion at large $J_{\mathrm{H}}$ is maximized when only one $e_{\mathrm{g}}$ electron is located at the JT center. Confirming this expectation, the double occupancy of a given orbital has been found to be negligible in previous investigations at intermediate and large values of the electron-phonon coupling, and in this situation, adding an on-site repulsion would not alter the physics of the state under investigation. Nevertheless, although the statements above are believed to be qualitatively correct, it should be checked explicitly in a more realistic model that indeed the physics found in the extreme case of only phononic interactions survives when both JT phononic and Coulombic interactions are considered. Thus, another purpose of this paper is to clarify the validity of the JT-phononic only model (large $J_{\mathrm{H}}$ ) by showing that the inclusion of the Coulomb interaction does not bring qualitative changes in the conclusions obtained from the purely phononic model. For this purpose, an analytic-numeric combination is employed, namely, the MFA including Coulombic terms is developed in detail and the results are compared with exact diagonalization data for a small cluster. In addition, the PS tendency is reexamined briefly within the framework of MFA, and some comments on previous results are provided. It is clear that addressing whether purely JT phononic or purely Coulombic approaches lead or not to 
qualitatively different phase diagrams is an important area of investigation.

As a special case of the general goal described in the previous paragraph, the issue of PS in the presence of Coulomb interactions will also be studied in this paper using computational techniques. In fact, it has not been analyzed whether models with two orbitals and Coulomb repulsions, i.e., without JT phonons, lead to mixed-phase tendencies as pure phononic models do. In this paper it is reported that a study of a simple 1D two-orbital model without phonons but with strong Coulomb interactions, produces PS tendencies similarly as reported in previous investigations by our group. ${ }^{11}$ Several features are in qualitative agreement with those observed using JT phonons, illustrating the similarities between the purely phononic and the purely Coulombic approaches to manganites, and the clear relevance of mixedphase characteristics for a proper description of these compounds. It is concluded that simple models for manganites, once studied with robust computational techniques, are able to reveal a complex phase diagram with several phases having characteristics quite similar to those observed experimentally.

The organization of the paper is as follows. In Sec. II, a theoretical model for manganites is introduced, and several reduced Hamiltonians are discussed in preparation for the subsequent investigations. Section III is devoted to MFA. The formulation for MFA is provided and a comparison with the exact diagonalization result is shown to discuss the validity of the MFA. It is argued that the JT-phononic interaction plays a role as relevant as the Coulombic interaction as suggested from the viewpoint of the continuity of the orbitalordered (OO) phase. In Sec. IV, as an application of the present MFA, the $\mathrm{CO} / \mathrm{OO}$ structure in half-doped manganites is studied. Especially, the origin of the CE-type state with the charge-stacked structure is discussed in detail. In Sec. V, the PS tendency is studied in the framework of the MFA for the purely JT phononic and Coulombic models. In Sec. VI, the two-orbital 1D Hubbard model is analyzed using the density matrix renormalization-group (DMRG) technique. Even in the purely Coulombic model, PS tendencies and CO phases are observed. Finally, in Sec. VII, the main results of the paper are summarized. The main overall conclusion is that studies based upon purely JT phononic (large $J_{\mathrm{H}}$ ) or Coulombic approaches do not differ substantially in their results, and there is no fundamental difference between them. For reasons to be described below, of the two approaches the best appears to be the phononic one due to its ability to select the proper orbital ordering pattern uniquely. PS appears in purely phononic and purely Coulombic approaches as well. In the Appendix, the relation between the PS scenarios for cuprates and manganites is discussed. Throughout this paper, units such that $\hbar=k_{\mathrm{B}}=1$ are used.

\section{MODELS FOR MANGANITES}

In order to explain the existence of ferromagnetism at low temperatures in manganites, the double-exchange mechanism has been usually invoked. ${ }^{3,4}$ In this framework, holes can improve their kinetic energy by polarizing the spins in their vicinity. If only one $e_{\mathrm{g}}$ orbital is used, the FM Kondo or one-orbital model is obtained, ${ }^{28}$ and several results for this model are already available in the literature. ${ }^{7-9}$ Although the PS tendencies have been identified in this context, it is clear that the one-orbital model is not sufficient to describe the rich physics of manganites, where the two active orbitals play a key role and the CO state close to density 0.5 is crucial for the appearance of a huge MR effect at this density. Thus, in this paper, the two-orbital model is discussed to understand the manganite physics, by highlighting the complementary roles of the JT phonon and Coulomb interactions.

\section{A. Hamiltonian}

Let us consider doubly degenerated $e_{\mathrm{g}}$ electrons, tightly coupled to localized $t_{2 \mathrm{~g}}$ spins and local distortions of the $\mathrm{MnO}_{6}$ octahedra. This situation is described by the Hamiltonian $H$ composed of five terms

$$
H=H_{\text {kin }}+H_{\text {Hund }}+H_{\mathrm{AFM}}+H_{\text {el-ph }}+H_{\text {el-el }} .
$$

The first term indicates the hopping motion of $e_{\mathrm{g}}$ electrons, given by

$$
H_{\mathrm{kin}}=-\sum_{\mathbf{i} \mathbf{\gamma} \gamma \gamma^{\prime} \sigma} t_{\gamma \gamma^{\prime}}^{\mathbf{a}} d_{\mathbf{i} \gamma \sigma}^{\dagger} d_{\mathbf{i}+\mathbf{a} \gamma^{\prime} \sigma}
$$

where $d_{\mathrm{ia} \sigma}\left(d_{\mathrm{ib} \sigma}\right)$ is the annihilation operator for an $e_{\mathrm{g}}$ electron with spin $\sigma$ in the $d_{x^{2}-y^{2}}\left(d_{3 z^{2}-r^{2}}\right)$ orbital at site $\mathbf{i}$, $\mathbf{a}$ is the vector connecting nearest-neighbor sites, and $t_{\gamma \gamma^{\prime}}^{\mathbf{a}}$ is the nearest-neighbor hopping amplitude between $\gamma$ and $\gamma^{\prime}$ orbitals along the a direction. The amplitudes are evaluated from the overlap integral between manganese and oxygen ions, ${ }^{29}$ given by

$$
t_{\mathrm{aa}}^{\mathbf{x}}=-\sqrt{3} t_{\mathrm{ab}}^{\mathbf{x}}=-\sqrt{3} t_{\mathrm{ba}}^{\mathbf{x}}=3 t_{\mathrm{bb}}^{\mathbf{x}}=t,
$$

for the $x$ direction,

$$
t_{\mathrm{aa}}^{\mathbf{y}}=\sqrt{3} t_{\mathrm{ab}}^{\mathrm{y}}=\sqrt{3} t_{\mathrm{ba}}^{\mathrm{y}}=3 t_{\mathrm{bb}}^{\mathbf{y}}=t,
$$

for the $y$ direction, and

$$
t_{\mathrm{bb}}^{\mathbf{z}}=4 t / 3, t_{\mathrm{aa}}^{\mathbf{z}}=t_{\mathrm{ab}}^{\mathbf{z}}=t_{\mathrm{ba}}^{\mathbf{z}}=0,
$$

for the $z$ direction. Note that $t_{\mathrm{aa}}^{\mathrm{x}}$ (equal to $t_{\mathrm{aa}}^{\mathrm{y}}$ by symmetry) is taken as the energy scale $t$. As for the value of $t$, it is estimated as $0.2-0.5 \mathrm{eV}^{30}$

The second term is the Hund coupling between the $e_{\mathrm{g}}$ electron spin $\mathbf{S}_{\mathbf{i}}$ and the localized $t_{2 \mathrm{~g}}$ spin $\mathbf{S}_{\mathbf{i}}$, given by

$$
H_{\text {Hund }}=-J_{\mathrm{H}} \sum_{\mathbf{i}} \mathbf{s}_{\mathbf{i}} \cdot \mathbf{S}_{\mathbf{j}},
$$

with $\mathbf{s}_{\mathbf{i}}=\Sigma_{\gamma \alpha \beta} d_{\mathbf{i} \gamma \alpha}^{\dagger} \boldsymbol{\sigma}_{\alpha \beta} d_{\mathbf{i} \gamma \beta}$, where $J_{\mathrm{H}}(>0)$ is the Hund coupling, and $\boldsymbol{\sigma}=\left(\sigma_{x}, \sigma_{y}, \sigma_{z}\right)$ are the Pauli matrices. Note here that the $t_{2 \mathrm{~g}}$ spins are assumed classical and normalized to $\left|\mathbf{S}_{\mathbf{i}}\right|=1$.

The third term accounts for the $G$-type AFM property of manganites in the fully hole doped limit, given by

$$
H_{\mathrm{AFM}}=J_{\mathrm{AF}} \sum_{\langle\mathbf{i}, \mathbf{j}\rangle} \mathbf{S}_{\mathbf{i}} \cdot \mathbf{S}_{\mathbf{j}},
$$


where $J_{\mathrm{AF}}$ is the AFM coupling between nearest-neighbor $t_{2 \mathrm{~g}}$ spins. The value of $J_{\mathrm{AF}}$ will be small in units of $t$ to make it compatible with experiments for the fully hole-doped $\mathrm{CaMnO}_{3}$ compound. ${ }^{31}$

In the fourth term, the coupling of $e_{\mathrm{g}}$ electrons to the lattice distortion is considered as ${ }^{32-34}$

$$
\begin{aligned}
H_{\mathrm{el}-\mathrm{ph}}= & g \sum_{\mathbf{i} \sigma}\left[Q_{1 \mathbf{i}}\left(d_{\mathbf{i} a \sigma}^{\dagger} d_{\mathbf{i} a \sigma}+d_{\mathbf{i} b \sigma}^{\dagger} d_{\mathbf{i} b \sigma}\right)+Q_{2 \mathbf{i}}\left(d_{\mathbf{i} a \sigma}^{\dagger} d_{\mathbf{i} b \sigma}\right.\right. \\
& \left.\left.+d_{\mathbf{i} b \sigma}^{\dagger} d_{\mathbf{i} a \sigma}\right)+Q_{3 \mathbf{i}}\left(d_{\mathbf{i} a \sigma}^{\dagger} d_{\mathbf{i} a \sigma}-d_{\mathbf{i} b \sigma}^{\dagger} d_{\mathbf{i} b \sigma}\right)\right] \\
& +(1 / 2) \sum_{\mathbf{i}}\left[k_{\mathrm{br}} Q_{1 \mathbf{i}}^{2}+k_{\mathrm{JT}}\left(Q_{2 \mathbf{i}}^{2}+Q_{3 \mathbf{i}}^{2}\right)\right]
\end{aligned}
$$

where $g$ is the coupling constant between $e_{\mathrm{g}}$ electrons and distortions of the $\mathrm{MnO}_{6}$ octahedron, $Q_{1 \mathrm{i}}$ is the breathingmode distortion, $Q_{2 \mathrm{i}}$ and $Q_{3 \mathrm{i}}$ are, respectively, the JT distortions for the $\left(x^{2}-y^{2}\right)$ - and $\left(3 z^{2}-r^{2}\right)$-type modes, and $k_{\mathrm{br}}$ $\left(k_{\mathrm{JT}}\right)$ is the spring constant for the breathing $(\mathrm{JT})$ mode distortions. Note here that the distortions are treated adiabatically ${ }^{35,36}$ and noncooperatively. ${ }^{37,38}$ An important energy scale characteristic of $H_{\mathrm{el}-\mathrm{ph}}$ is the static JT energy, defined by

$$
E_{\mathrm{JT}}=g^{2} /\left(2 k_{\mathrm{JT}}\right),
$$

which is naturally obtained by the scaling of $Q_{\mu \mathbf{i}}$ $=\left(g / k_{\mathrm{JT}}\right) q_{\mu \mathbf{i}}$ with $\mu=1,2$, and 3 , where $g / k_{\mathrm{JT}}$ is the typical length scale for the JT distortion. By using $E_{\mathrm{JT}}$ and $t$, it is convenient to introduce the nondimensional electron-phonon coupling constant $\lambda$ as $\lambda=\sqrt{2 E_{\mathrm{JT}} / t}$. The characteristic energy for the breathing-mode distortion is given by $E_{\mathrm{br}}$ $=g^{2} /\left(2 k_{\mathrm{br}}\right)=E_{\mathrm{JT}} / \beta$ with $\beta=k_{\mathrm{br}} / k_{\mathrm{JT}}$.

The last term indicates the Coulomb interactions between $e_{\mathrm{g}}$ electrons, expressed by

$$
\begin{aligned}
H_{\mathrm{el}-\mathrm{el}}= & U \sum_{\mathbf{i} \gamma} \rho_{\mathbf{i} \gamma \uparrow} \rho_{\mathbf{i} \gamma \downarrow}+U^{\prime} \sum_{\mathbf{i} \sigma \sigma^{\prime}} \rho_{\mathbf{i} a \sigma} \rho_{\mathbf{i b} \sigma^{\prime}} \\
& +J \sum_{\mathbf{i} \sigma \sigma^{\prime}} d_{\mathbf{i} a \sigma}^{\dagger} d_{\mathbf{i b} \sigma^{\prime}}^{\dagger} d_{\mathbf{i} a \sigma^{\prime}} d_{\mathbf{i} b \sigma}+V \sum_{\langle\mathbf{i}, \mathbf{j}\rangle} \rho_{\mathbf{i}} \rho_{\mathbf{j}},
\end{aligned}
$$

with $\rho_{\mathbf{i} \gamma \sigma}=d_{\mathbf{i} \gamma \sigma}^{\dagger} d_{\mathbf{i} \gamma \sigma}$ and $\rho_{\mathbf{i}}=\Sigma_{\gamma \sigma} \rho_{\mathbf{i} \gamma \sigma}$, where $U$ is the intraorbital Coulomb interaction, $U^{\prime}$ is the interorbital Coulomb interaction, $J$ is the interorbital exchange interaction, and $V$ is the nearest-neighbor Coulomb interaction. Note that the Hund-coupling term $H_{\text {Hund }}$ between conduction and localized spins also arises from Coulombic effects, but in this paper, the "Coulomb interaction" refers to the direct electrostatic repulsion between $e_{\mathrm{g}}$ electrons. Note also that the relation $U=U^{\prime}+2 J$ holds in the localized ion system. Although the validity of this relation may not be guaranteed in the actual material, it is assumed to hold in this paper to reduce the parameter space in the calculation.

\section{B. Reduced Hamiltonians}

The Hamiltonian Eq. (1) is believed to define an appropriate model for manganites, but it is quite difficult to solve it exactly. In order to investigate further the properties of manganites based on $H$, some simplifications and approximations are needed. A simplification without the loss of es-

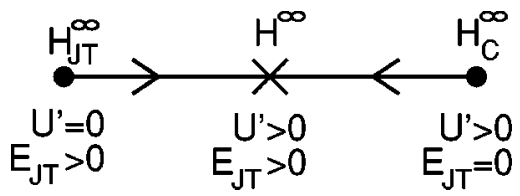

FIG. 1. Schematic representation of the two approaches (JahnTeller and Coulombic) to the realistic situation based on the simplified model $H^{\infty}$.

sential physics is to take the widely used limit $J_{\mathrm{H}}=\infty .{ }^{39} \mathrm{In}$ such a limit, the $e_{\mathrm{g}}$ electron spin perfectly aligns along the $t_{2 \mathrm{~g}}$ spin direction, reducing the number of degrees of freedom. Moreover, there is a clear advantage that both the intraorbital on-site term and the exchange term can be neglected in $H_{\mathrm{el}-\mathrm{el}}$. Thus, the following simplified model is obtained:

$$
\begin{aligned}
& H^{\infty}=-\sum_{\mathbf{i} \gamma \gamma \gamma^{\prime}} \mathcal{S}_{\mathbf{i}, \mathbf{i}+\mathbf{a}{ }^{\prime}{ }_{\gamma \gamma^{\prime}}} c_{\mathbf{i} \gamma}^{\dagger} c_{\mathbf{i}+\mathbf{a} \gamma^{\prime}}+J_{\mathrm{AF}} \sum_{\langle\mathbf{i}, \mathbf{j}\rangle} \mathbf{S}_{\mathbf{i}} \cdot \mathbf{S}_{\mathbf{j}} \\
& +E_{\mathrm{JT}} \sum_{\mathbf{i}}\left[2\left(q_{1 \mathbf{i}} n_{\mathbf{i}}+q_{2 \mathbf{i}} \tau_{x \mathbf{i}}+q_{3 \mathbf{i}} \tau_{z \mathbf{i}}\right)+\beta q_{1 \mathbf{i}}^{2}+q_{2 \mathbf{i}}^{2}+q_{3 \mathbf{i}}^{2}\right] \\
& +U^{\prime} \sum_{\mathbf{i}} n_{\mathbf{i a}} n_{\mathbf{i b}}+V \sum_{\langle\mathbf{i}, \mathbf{j}\rangle} n_{\mathbf{i}} n_{\mathbf{j}},
\end{aligned}
$$

where $c_{\mathbf{i} \gamma}$ is the spinless $e_{\mathrm{g}}$ electron operator, given by ${ }^{40}$ $c_{\mathbf{i} \gamma}=\cos \left(\theta_{\mathbf{i}} / 2\right) d_{\mathbf{i} \gamma \uparrow}+\sin \left(\theta_{\mathbf{i}} / 2\right) e^{-i \phi_{\mathbf{i}}} d_{\mathbf{i} \gamma \downarrow}$, the angles $\theta_{\mathbf{i}}$ and $\phi_{\mathbf{i}}$ define the direction of the classical $t_{2 \mathrm{~g}}$ spin at site $\mathbf{i}, n_{\mathbf{i} \gamma}$ $=c_{\mathbf{i} \gamma}^{\dagger} c_{\mathbf{i} \gamma}, \quad n_{\mathbf{i}}=\Sigma_{\gamma} n_{\mathbf{i} \gamma}, \quad \tau_{x \mathbf{i}}=c_{\mathbf{i} a}^{\dagger} c_{\mathbf{i} b}+c_{\mathbf{i} b}^{\dagger} c_{\mathbf{i} a}$, and $\tau_{z \mathbf{i}}=c_{\mathbf{i} a}^{\dagger} c_{\mathbf{i} a}$ $-c_{\mathrm{i} b} c_{\mathrm{i} b}$. Here $\mathcal{S}_{\mathbf{i}, \mathbf{j}}$ denotes the change of hopping amplitude due to the difference in angles between $t_{2 \mathrm{~g}}$ spins at sites $\mathbf{i}$ and $\mathbf{j}$, given by $\mathcal{S}_{\mathbf{i}, \mathbf{j}}=\cos \left(\theta_{\mathbf{i}} / 2\right) \cos \left(\theta_{\mathbf{j}} / 2\right)$ $+\sin \left(\theta_{\mathbf{i}} / 2\right) \sin \left(\theta_{\mathbf{j}} / 2\right) e^{-i\left(\phi_{\mathbf{i}}-\phi_{\mathbf{j}}\right)}$. In principle, $\theta_{\mathbf{i}}$ and $\phi_{\mathbf{i}}$ should be optimized to provide the lowest-energy state. However, in the following analytic approach, only spin configurations such that nearest-neighbor $t_{2 \mathrm{~g}}$ spins are either FM or AFM will be considered. Namely, the spin canting state is excluded from the outset. The validity of this simplification is later checked by comparison with the numerical results obtained from a relaxation technique. Note that this simplification does not restrict us to only fully FM or $G$-type AFM states, but a wide variety of other states, such as the CE-type one, can also be studied.

Based on the reduced Hamiltonian $H^{\infty}$, it is possible to consider two scenarios for manganites, as schematically shown in Fig. 1. Temporarily, $V$ will not be taken into account to focus on the competition between $E_{\mathrm{JT}}$ and $U^{\prime}$. One of them is the JT-phonon scenario in which $U^{\prime}$ is considered as a correction into the purely JT-phononic model $H_{\mathrm{JT}}^{\infty}$ $=H^{\infty}\left(U^{\prime}=0, V=0\right)$ taken as starting point. Another is the Coulomb scenario where the JT distortion is a correction into the purely Coulombic Hubbard-like model $H_{\mathrm{C}}^{\infty}=H^{\infty}\left(E_{\mathrm{JT}}\right.$ $=0)$. Of course, if the many-body analysis were very accurate then there should be no difference between the final conclusions obtained from both scenarios when the realistic situation, $E_{\mathrm{JT}}>0$ and $U^{\prime}>0$, is approached. However, if this realistic situation can be continuously obtained from the limiting cases $U^{\prime}=0$ or $E_{\mathrm{JT}}=0$, then it is enough to consider only the limiting models $H_{\mathrm{JT}}^{\infty}$ or $H_{\mathrm{C}}^{\infty}$ to grasp the essence of 
the manganite physics. On the other hand, it may also occur that starting in one of the two extreme cases a singularity exists preventing a smooth continuation into the realistic region of parameters. In this case, just one of the scenarios would be valid. For investigations in this context, the simplified model $H^{\infty}$ certainly provides a good stage to examine the roles of $U^{\prime}$ and $E_{\mathrm{JT}}$. In the following section, this point will be discussed in detail.

Another possible simplification could have been obtained by neglecting the electron-electron interaction in $H$ but keeping the Hund coupling finite (but large), leading to the following purely JT-phononic model with active spin degrees of freedom: ${ }^{11,38} H_{\mathrm{JT}}=H_{\mathrm{kin}}+H_{\mathrm{Hund}}+H_{\mathrm{AFM}}+H_{\mathrm{el}-\mathrm{ph}}$. Note that this model is reduced to $H_{\mathrm{JT}}^{\infty}$ when the limit $J_{\mathrm{H}}=\infty$ is taken. To solve $H_{\mathrm{JT}}$, numerical methods such as Monte Carlo techniques and the relaxation method have been employed in the past. ${ }^{11,38}$ However, here this model will not be discussed explicitly, but the simplified version $H_{\mathrm{JT}}^{\infty}$ will be investigated in the next section. Qualitatively, the negligible values of the probability of double occupancy at large $\lambda$ justifies the neglect of $H_{\mathrm{el}-\mathrm{el}}$. In addition, the results of recent studies addressing the $A$-type AFM phase of the holeundoped limit using cooperative JT phonons have been shown to be quite similar to those found with pure Coulombic interactions, ${ }^{38}$ the latter treated with the MFA.

Nevertheless, in spite of the above discussed indications that the JT and Coulomb formalisms lead to similar physics, it would be important to verify this belief by studying a multiorbital model with only Coulombic terms, without the extra approximation of using mean-field techniques for its analysis. Of particular relevance is whether PS tendencies and charge ordering appear in this case, as they do in the JT-phononic model. This analysis is particularly important since, as explained before, a mixture of phononic and Coulombic interactions is expected to be needed for a proper quantitative description of manganites. For this purpose, yet another simplified model will be analyzed in this paper:

$$
H_{\mathrm{C}}=H_{\text {kin }}+H_{\mathrm{el}-\mathrm{el}} \cdot
$$

Note that this model cannot be exactly reduced to $H_{\mathrm{C}}^{\infty}$, since the Hund coupling term between $e_{\mathrm{g}}$ electrons and $t_{2 \mathrm{~g}}$ spins is not explicitly included. The reason for this extra simplification is that the numerical complexity in the analysis of the model is drastically reduced by neglecting the localized $t_{2 \mathrm{~g}}$ spins. In the FM phase, this is an excellent approximation but not necessarily for other magnetic arrangements. Nevertheless the authors believe that it is important to establish with accurate numerical techniques whether the PS tendencies are already present in this simplified two-orbital models with Coulomb interactions, even if not all degrees of freedom are incorporated from the outset. Adding the $S=3 / 2$ quantum localized spins to the problem would considerably increase the size of the Hilbert space of the model, making it intractable with current computational techniques.

\section{MEAN-FIELD APPROXIMATION FOR $\boldsymbol{H}^{\infty}$}

Even a simplified model such as $H^{\infty}$ is still difficult to be solved exactly except for some special cases. Thus, in this section, the MFA is developed for $H^{\infty}$ to attempt to grasp its essential physics. Note that even at the mean-field level, due
TABLE I. Phase $\xi_{\mathrm{i}}$ and the corresponding $e_{\mathrm{g}}$-electron orbitals.

\begin{tabular}{lcc}
\hline \hline \multicolumn{1}{c}{$\xi_{\mathbf{i}}$} & a orbital & b orbital \\
\hline 0 & $x^{2}-y^{2}$ & $3 z^{2}-r^{2}$ \\
$\pi / 3$ & $3 y^{2}-r^{2}$ & $z^{2}-x^{2}$ \\
$2 \pi / 3$ & $y^{2}-z^{2}$ & $3 x^{2}-r^{2}$ \\
$\pi$ & $3 z^{2}-r^{2}$ & $x^{2}-y^{2}$ \\
$4 \pi / 3$ & $z^{2}-x^{2}$ & $3 y^{2}-r^{2}$ \\
$5 \pi / 3$ & $3 x^{2}-r^{2}$ & $y^{2}-z^{2}$ \\
\hline
\end{tabular}

care should be paid to the self-consistent treatment to lift the double degeneracy in $e_{g}$ electrons.

\section{A. Formulation}

First let us rewrite the electron-phonon term by applying a simple standard decoupling such as $q_{2 \mathrm{i}} \tau_{x \mathrm{i}} \approx\left\langle q_{2 \mathrm{i}}\right\rangle \tau_{x \mathrm{i}}$ $+q_{2 \mathbf{i}}\left\langle\tau_{x \mathbf{i}}\right\rangle-\left\langle q_{2 \mathrm{i}}\right\rangle\left\langle\tau_{x \mathbf{i}}\right\rangle$, where the bracket means the average value using the mean-field Hamiltonian described below. By minimizing the phonon energy, the local distortion is determined in the MFA as $q_{1 \mathbf{i}}=-\left\langle n_{\mathbf{i}}\right\rangle / \beta, q_{2 \mathbf{i}}=-\left\langle\tau_{x \mathbf{i}}\right\rangle$, and $q_{3 \mathbf{i}}$ $=-\left\langle\tau_{z \mathbf{i}}\right\rangle$. Thus, the electron-phonon term in the MFA is given by

$$
\begin{aligned}
H_{\mathrm{el}-\mathrm{ph}}^{\mathrm{MF}}= & -2 \sum_{\mathbf{i}}\left[E_{\mathrm{br}}\left\langle n_{\mathbf{i}}\right\rangle n_{\mathbf{i}}+E_{\mathrm{JT}}\left(\left\langle\tau_{x \mathbf{i}}\right\rangle \tau_{x \mathbf{i}}+\left\langle\tau_{z \mathbf{i}}\right\rangle \tau_{z \mathbf{i}}\right)\right] \\
& +\sum_{\mathbf{i}}\left[E_{\mathrm{br}}\left\langle n_{\mathbf{i}}\right\rangle^{2}+E_{\mathrm{JT}}\left(\left\langle\tau_{x \mathbf{i}}\right\rangle^{2}+\left\langle\tau_{z \mathbf{i}}\right\rangle^{2}\right)\right] .
\end{aligned}
$$

Now let us turn our attention to the electron-electron interaction term. At a first glance, it appears enough to make a similar decoupling procedure for $H_{\mathrm{el}-\mathrm{el}}$. However, such a decoupling cannot be uniquely carried out, since $H_{\mathrm{el}-\mathrm{el}}$ is invariant with respect to the choice of $e_{g}$-electron orbitals due to the local SU(2) symmetry in the orbital space. Thus, to obtain the $\mathrm{OO}$ state, it is necessary to find the optimal orbital set by determining the relevant $e_{\mathrm{g}}$-electron orbital self-consistently at each site. For this purpose, it is useful to express $q_{2 \mathbf{i}}$ and $q_{3 \mathbf{i}}$ in polar coordinates as

$$
q_{2 \mathrm{i}}=q_{\mathbf{i}} \sin \xi_{\mathbf{i}}, \quad q_{3 \mathbf{i}}=q_{\mathbf{i}} \cos \xi_{\mathbf{i}},
$$

where $q_{\mathbf{i}}$ and $\xi_{\mathbf{i}}$ are, respectively, determined as $q_{\mathbf{i}}$ $=\sqrt{\left\langle\tau_{x \mathrm{i}}\right\rangle^{2}+\left\langle\tau_{z \mathrm{i}}\right\rangle^{2}}$ and $\xi_{\mathrm{i}}=\pi+\tan ^{-1}\left(\left\langle\tau_{x \mathrm{i}}\right\rangle /\left\langle\tau_{z \mathrm{i}}\right\rangle\right)$ in the MFA. By using the phase $\xi_{\mathrm{i}}, c_{\mathrm{ia}}$ and $c_{\mathrm{ib}}$ are transformed into $\tilde{c}_{\mathrm{ia}}$ and $\tilde{c}_{\mathrm{ib}}$ as $\tilde{c}_{\mathrm{ia}}=e^{i \xi_{\mathrm{i}} / 2}\left[c_{\mathrm{ia}} \cos \left(\xi_{\mathrm{i}} / 2\right)+c_{\mathrm{ib}} \sin \left(\xi_{\mathrm{i}} / 2\right)\right]$ and $\tilde{c}_{\mathrm{ib}}$ $=e^{i \xi_{\mathrm{i}} / 2}\left[-c_{\mathrm{ia}} \sin \left(\xi_{\mathrm{i}} / 2\right)+c_{\mathrm{ib}} \cos \left(\xi_{\mathrm{i}} / 2\right)\right]$. Note that the phase factor is needed for the assurance of the single-valuedness of the basis function, leading to the molecular Aharonov-Bohm effect. ${ }^{19}$

It should be noted that the phase $\xi_{\mathrm{i}}$ determines the electron orbital set at each site. For instance, at $\xi_{\mathrm{i}}=2 \pi / 3$, a and b denote the $d_{y^{2}-z^{2}}$ and $d_{3 x^{2}-r^{2}}$ orbital, respectively. In Table I, the correspondence between $\xi_{\mathbf{i}}$ and the local orbital is summarized for several important values of $\xi_{\mathrm{i}}$. Note here that $d_{3 x^{2}-r^{2}}$ and $d_{3 y^{2}-r^{2}}$ never appear as the local orbital set. In recent publications ${ }^{41}$ those were inadvertently treated as an orthogonal orbital set to reproduce the experimental results, but such a treatment is essentially incorrect, since the 
orbital ordering is not due to the simple alternation of two arbitrary kinds of orbitals, as shown in the following discussion.

By the above transformation, $H_{\mathrm{el}-\mathrm{ph}}^{\mathrm{MF}}$ and $H_{\mathrm{el}-\mathrm{el}}$ are, respectively, rewritten as

$$
\begin{aligned}
H_{\mathrm{el}-\mathrm{ph}}^{\mathrm{MF}}= & \sum_{\mathbf{i}}\left\{E_{\mathrm{br}}\left(-2\left\langle n_{\mathbf{i}}\right\rangle \tilde{n}_{\mathbf{i}}+\left\langle n_{\mathbf{i}}\right\rangle^{2}\right)\right. \\
& \left.+E_{\mathrm{JT}}\left[2 q_{\mathbf{i}}\left(\tilde{n}_{\mathrm{ia}}-\tilde{n}_{\mathrm{ib}}\right)+q_{\mathbf{i}}^{2}\right]\right\},
\end{aligned}
$$

and

$$
H_{\mathrm{el}-\mathrm{el}}=U^{\prime} \sum_{\mathbf{i}} \tilde{n}_{\mathbf{i a}} \tilde{n}_{\mathbf{i b}}+V \sum_{\langle\mathbf{i}, \mathbf{j}\rangle} \tilde{n}_{\mathbf{i}} \tilde{n}_{\mathbf{j}},
$$

where $\tilde{n}_{\mathbf{i} \gamma}=\tilde{c}_{\mathbf{i} \gamma}^{\dagger} \tilde{c}_{\mathbf{i} \gamma}$ and $\tilde{n}_{\mathbf{i}}=\tilde{n}_{\mathbf{i} \mathbf{a}}+\tilde{n}_{\mathbf{i b}}$. Note that $H_{\text {el-el }}$ is invariant with respect to the choice of $\xi_{\mathbf{i}}$. Now let us apply the decoupling procedure as $\tilde{n}_{\mathrm{ia}} \tilde{n}_{\mathrm{ib}} \approx\left\langle\tilde{n}_{\mathrm{ia}}\right\rangle \tilde{n}_{\mathrm{ib}}+\tilde{n}_{\mathrm{ia}}\left\langle\tilde{n}_{\mathrm{ib}}\right\rangle$ $-\left\langle\tilde{n}_{\mathbf{i a}}\right\rangle\left\langle\tilde{n}_{\mathrm{ib}}\right\rangle$, by noting the relations $\left\langle\tilde{n}_{\mathbf{i a}}\right\rangle=\left(\left\langle n_{\mathbf{i}}\right\rangle-q_{\mathrm{i}}\right) / 2$, $\left\langle\tilde{n}_{\mathrm{ib}}\right\rangle=\left(\left\langle n_{\mathrm{i}}\right\rangle+q_{\mathrm{i}}\right) / 2$, and $n_{\mathbf{i}}=\tilde{n}_{\mathbf{i}}$. Then, the electron-electron interaction term is given in the MFA as

$$
\begin{aligned}
H_{\mathrm{el}-\mathrm{el}}^{\mathrm{MF}}= & \left(U^{\prime} / 4\right) \sum_{\mathbf{i}}\left[2\left\langle n_{\mathbf{i}}\right\rangle \tilde{n}_{\mathbf{i}}-\left\langle n_{\mathbf{i}}\right\rangle^{2}+2 q_{\mathbf{i}}\left(\tilde{n}_{a \mathbf{i}}-\tilde{n}_{b \mathbf{i}}\right)+q_{\mathbf{i}}^{2}\right] \\
& +V \sum_{\mathbf{i a}}\left[\left\langle n_{\mathbf{i}+\mathbf{a}}\right\rangle \tilde{n}_{\mathbf{i}}-(1 / 2)\left\langle n_{\mathbf{i}+\mathbf{a}}\right\rangle\left\langle n_{\mathbf{i}}\right\rangle\right] .
\end{aligned}
$$

By combining $H_{\mathrm{el}-\mathrm{ph}}^{\mathrm{MF}}$ with $H_{\mathrm{el}-\mathrm{el}}^{\mathrm{MF}}$ and transforming $\tilde{c}_{\mathrm{ia}}$ and $\tilde{c}_{\mathrm{ib}}$ into the original operators as $c_{\mathrm{ia}}$ and $c_{\mathrm{ib}}$, the mean-field Hamiltonian is finally obtained as

$$
\begin{aligned}
H_{\mathrm{MF}}^{\infty}= & -\sum_{\mathbf{i} \mathbf{a} \gamma \gamma^{\prime}} t_{\gamma \gamma^{\prime}}^{\mathbf{a}} c_{\mathbf{i} \gamma}^{\dagger} c_{\mathbf{i}+\mathbf{a} \gamma^{\prime}}+J_{\mathrm{AF}} \sum_{\langle\mathbf{i}, \mathbf{j}\rangle} \mathbf{S}_{\mathbf{i}} \cdot \mathbf{S}_{\mathbf{j}} \\
& +\widetilde{E}_{\mathrm{JT}} \sum_{\mathbf{i}}\left[-2\left(\left\langle\tau_{x \mathbf{i}}\right\rangle \tau_{x \mathbf{i}}+\left\langle\tau_{z \mathbf{i}}\right\rangle \tau_{z \mathbf{i}}\right)+\left\langle\tau_{x \mathbf{i}}\right\rangle^{2}+\left\langle\tau_{z \mathbf{i}}\right\rangle^{2}\right] \\
& +\sum_{\mathbf{i}}\left[\left(\widetilde{U}^{\prime} / 2\right)\left\langle n_{\mathbf{i}}\right\rangle+V \sum_{\mathbf{a}}\left\langle n_{\mathbf{i}+\mathbf{a}}\right\rangle\right] n_{\mathbf{i}} \\
& -\sum_{\mathbf{i}}\left[\left(\widetilde{U}^{\prime} / 4\right)\left\langle n_{\mathbf{i}}\right\rangle+(V / 2) \sum_{\mathbf{a}}\left\langle n_{\mathbf{i}+\mathbf{a}}\right\rangle\right]\left\langle n_{\mathbf{i}}\right\rangle
\end{aligned}
$$

where the renormalized JT energy is given by

$$
\widetilde{E}_{\mathrm{JT}}=E_{\mathrm{JT}}+U^{\prime} / 4,
$$

and the renormalized interorbital Coulomb interaction is expressed as

$$
\widetilde{U}^{\prime}=U^{\prime}-4 E_{\mathrm{br}} .
$$

Physically, the former relation indicates that the JT energy is effectively enhanced by $U^{\prime}$. Namely, the strong on-site Coulombic correlation plays the same role as that of the JT phonon, at least at the mean-field level, indicating that it is not necessary to include $U^{\prime}$ explicitly in the models, as has been emphasized by the present authors in several publications. The latter equation for $\widetilde{U}^{\prime}$ means that the one-site interorbital Coulomb interaction is effectively reduced by the breathing- mode phonon, since the optical-mode phonon provides an effective attraction between electrons. The expected positive value of $\widetilde{U}^{\prime}$ indicates that $e_{\mathrm{g}}$ electrons dislike double occupancy at the site, since the energy loss is proportional to the average local electron number in the mean-field argument. Thus, to exploit the gain due to the static JT energy and avoid the loss due to the on-site repulsion, an $e_{\mathrm{g}}$ electron will singly occupy the site. Note that $\beta$ is suggested to be larger than unity in manganites. ${ }^{36,42}$ It has been shown that the breathing-model distortion is suppressed for reasonable parameter choice, even if it is included in the calculation. Thus, in the following, $\beta$ is taken to be infinity for simplicity.

\section{B. JT-phononic vs Coulombic scenario}

In order to check the validity of the present mean-field treatment, it is necessary to compare the mean-field result with some exact solutions. For this purpose, numerical techniques are here applied in small-size clusters. For a set of lattice distortions, $\left\{q_{1 \mathrm{i}}, q_{2 \mathrm{i}}, q_{3 \mathrm{i}}\right\}$, the ground-state energy is calculated by using the exact diagonalization method to include exactly the effect of electron correlations. By searching for the minimum energy, the set of optimal distortions $\left\{q_{1 \mathbf{i}}^{\mathrm{opt}}, q_{2 \mathbf{i}}^{\mathrm{opt}}, q_{3 \mathbf{i}}^{\mathrm{opt}}\right\}$ is determined. In this exact treatment, the phase to characterize the local orbital set is defined by $\xi_{\mathrm{i}}$ $=\tan ^{-1}\left(q_{2 \mathbf{i}}^{\mathrm{opt}} / q_{3 \mathbf{i}}^{\mathrm{opt}}\right)$.

In this subsection, a small four-site 1D chain with the periodic boundary condition is used in order to grasp the most basic aspects of the problem with a minimum requirement of CPU time. The small cluster size and the low dimensionality will be severe tests for the MFA. However, if the validity is verified under such severe conditions, the meanfield result can be more easily accepted in the large cluster limit and in higher dimensions. In order to focus our attention on the roles of JT phonon and on-site correlations, the $t_{2 \mathrm{~g}}$ spin configuration is assumed to be ferromagnetic (thus, $J_{\mathrm{AF}}$ will not play an important role) and $V$ is set as zero. The effect of $V$ will be discussed in the next subsection in the context of the $\mathrm{CO}$ state of half-doped manganites. The realistic hopping matrix set is used for the $1 \mathrm{D}$ chain in the $x$ direction, although the conclusions are independent of the direction of the $1 \mathrm{D}$ chain.

In Figs. 2(a)-2(f), the results for $n=1$ and $E_{\mathrm{JT}}=t$ are shown. Figure 2(a) contains the ground-state energy plotted as a function of $U^{\prime}$ for a fixed value of $E_{\mathrm{JT}}$. In general, the many-body effects cannot be perfectly included within the MFA. However, when $U^{\prime}$ is introduced to the 1D chain with $E_{\mathrm{JT}} \neq 0$, the results become exact due to the special properties of one dimension and the use of the realistic hopping amplitude ${ }^{43}$ Namely, the hopping direction is restricted only along one axis, and the orbital set is uniquely determined due to the optimization of the JT distortion. In such an orbital polarized situation, the electron correlation is included exactly even in the mean-field level. Unfortunately, for 2D or 3D FM phases, there is no guarantee that the MFA provides the exact results, as discussed briefly in the final paragraph, but the MFA for the Coulomb interactions is still expected to provide the qualitatively correct tendency due to the general expectation that the MFA becomes valid in higher dimensions. ${ }^{44}$ However, in the purely JT-phononic model $H_{\mathrm{JT}}^{\infty}$, a remarkable fact appears. Namely, the MFA is always 


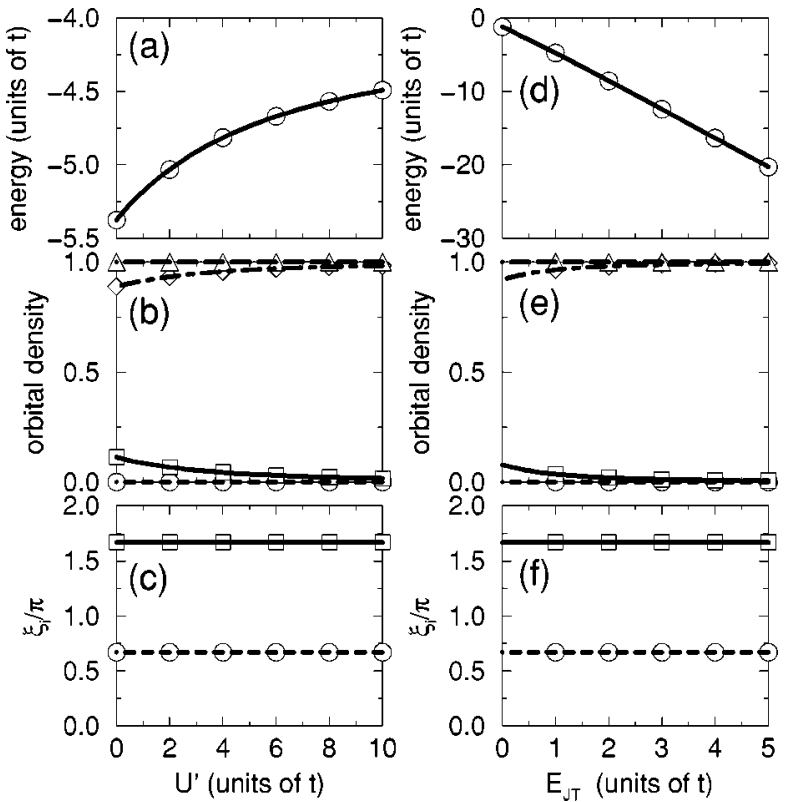

FIG. 2. Mean-field and exact-diagonalization results at $n=1$ for a four-site 1D chain with the realistic hopping amplitude along the $x$ axis. In all figures, the curves denote the mean-field results and open symbols indicate the results obtained by the combination of the exact diagonalization and the relaxation method in the phonons. Figures 2(a)-2(c) indicate the $U^{\prime}$ dependence of some quantities for the special case $E_{\mathrm{JT}}=t$, while these same quantities are plotted as a function of $E_{\mathrm{JT}}$ for $U^{\prime}=5 t$ in Figs. 2(d)-2(f). In (a) and (d), ground-state energies are shown. In (b) and (e), orbital densities, $\left\langle\tilde{n}_{\mathbf{i a}}\right\rangle$ and $\left\langle\tilde{n}_{\mathbf{i b}}\right\rangle$, are plotted. The solid curve and open square denote $\left\langle\tilde{n}_{\mathrm{ia}}\right\rangle$ for site 1 . The broken curve and open circle denote $\left\langle\tilde{n}_{\mathrm{ia}}\right\rangle$ for site 2 . The dash-broken curve and open diamond indicate $\left\langle\tilde{n}_{\mathrm{ib}}\right\rangle$ for site 1 . The long-dashed curve and open triangle indicate $\left\langle\tilde{n}_{\mathrm{ib}}\right\rangle$ for site 2 . In (c) and (f), the phases $\xi_{\mathbf{i}}$ are plotted. The solid line and open square denote $\xi_{\mathbf{i}}$ for site 1 . The broken curve and open circle denote $\xi_{\mathbf{i}}$ for site 2 . Note that only the results at site 1 and 2 are depicted, since the results at site 3 and 4 are the same as those at site 1 and 2 , respectively.

exact irrespective of the cluster size, electron density, and dimensionality in $H_{\mathrm{JT}}^{\infty}$. This is quite natural, since the static distortion gives only the potential energy for the $e_{\mathrm{g}}$ electrons, and this fermionic sector is essentially a one-body problem, even though the potential should be optimized selfconsistently. The lattice distortion is basically determined by the local electron density, and the MFA becomes exact in the static distortion limit.

For the reasons discussed above the MFA works quite well in the JT scenario, but this fact does not mean that the obtained state is trivial, since the orbital degree of freedom is active and several nontrivial $\mathrm{OO}$ phases occur. To visualize this result, the orbital densities, $\left\langle\tilde{n}_{\mathbf{i a}}\right\rangle$ and $\left\langle\tilde{n}_{\mathbf{i b}}\right\rangle$, are shown in Fig. 2(b), and the phase $\xi_{\mathrm{i}}$ is plotted in Fig. 2(c). It is observed that $\left\langle\tilde{n}_{\mathbf{i a}}\right\rangle$ becomes very small and $\left\langle\tilde{n}_{\mathbf{i b}}\right\rangle$ is almost unity for all the sites. For even and odd sites, $\xi_{\mathbf{i}}$ is given by $2 \pi / 3$ and $5 \pi / 3$, respectively. Namely, the occupied b orbital is $d_{3 x^{2}-r^{2}}$ at the even sites and $d_{y^{2}-z^{2}}$ at the odd sites, respectively. This is simply the orbital-staggered state in the spin FM phase, which has been observed in the Monte Carlo analysis. ${ }^{11}$ The mechanism of its occurrence is quite simple:
Imagine two adjacent sites in which one $e_{\mathrm{g}}$ electron is present at each site. If the limit $E_{\mathrm{JT}} \gg t$ is considered, the energy gain can be evaluated in second order with respect to $t$, and this energy is maximized when the occupied orbital in a certain site is exactly the same as the unoccupied orbital in the neighboring site. Namely, the difference in $\xi_{\mathrm{i}}$ between two adjacent sites should be $\pi$ at $n=1$. Note that the value of $\xi_{\mathrm{i}}$ depends on the hopping direction.

Next let us examine the Coulombic scenario, still using a small four-site cluster to compare mean-field results against exact ones. In Figs. 2(d)-2(f), the ground-state energy, orbital densities, and the phase are plotted as a function of $E_{\mathrm{JT}}$ for a fixed value of $U^{\prime}=5 t$. Again it can be observed that the MFA works quite well, except for the case of $E_{\mathrm{JT}}=0$. In this pure Coulombic limit, the JT distortion does not occur, and thus, the results are obtained only by the exact diagonalization for $H_{\mathrm{C}}^{\infty}$. The ground-state energy obtained in the MFA converges to the exact result in the limit of $E_{\mathrm{JT}} \rightarrow 0$. However, no symbols are shown at $E_{\mathrm{JT}}=0$ in Figs. 2(e) and 2(f), since the orbital densities and the phase could not be fixed at $E_{\mathrm{JT}}=0$. This is due to the fact that the energy is invariant for any choice of local orbital, indicating that $\left\langle\tilde{n}_{\mathrm{ia}}\right\rangle,\left\langle\tilde{n}_{\mathrm{ib}}\right\rangle$, and $\xi_{\mathrm{i}}$ cannot be determined at each site for $E_{\mathrm{JT}}=0$. In other words, the $\mathrm{OO}$ state is not uniquely fixed within the purely Coulombic model $H_{\mathrm{C}}^{\infty}$ in the sense that the special orbital is not specified at each site. ${ }^{45}$ However, if the orbital correlation function is examined, it is possible to determine the orbital pattern in the $\mathrm{OO}$ phase even in the simplified Coulombic model. In fact, the orbital staggered tendency at $n=1$ is detected when the orbital correlation as a function of distance is studied, as will be discussed in Sec. VI.

Now the roles of the JT phonon and on-site correlation are discussed. At finite electron-phonon coupling, the optimized orbital is determined, and the MFA provides an essentially exact result for the shape of the orbital. Note that carrying out unbiased Monte Carlo simulations and using the relaxation technique are still important tasks, since the present MFA works quite well only for a fixed $t_{2 \mathrm{~g}}$ spin background. Thus, it is an unavoidable step to check whether the assumed $t_{2 \mathrm{~g}}$ spin pattern is really stable or not with the use of unbiased techniques by optimizing the lattice distortions as well as the $t_{2 \mathrm{~g}}$ spin directions simultaneously. In this sense, the analytic mean-field approach becomes very powerful when it is combined with numerical unbiased techniques.

In the purely Coulombic model, however, no unique optimal orbital is determined at each site from the energetical viewpoint due to the local $\mathrm{SU}(2)$ symmetry, although it can be deduced from the orbital correlation function. Only when the JT distortion is included, the optimal orbital set at each site is uniquely determined from the competition between the kinetic energy gain and the potential loss due to the lattice distortion. As a consequence, it appears that the natural starting model to understand the properties of manganites should be the JT-phononic model, and it is enough to include the effect of $U^{\prime}$ on $H_{\mathrm{JT}}^{\infty}$. Although the validity of this statement has been shown in a limited situation in this paper, it is believed to be correct in other cases. On the other hand, if the starting model is chosen as the purely Coulombic model, the degeneracy in the orbital space is lifted when the JT distortion is introduced, indicating that the ground-state property 


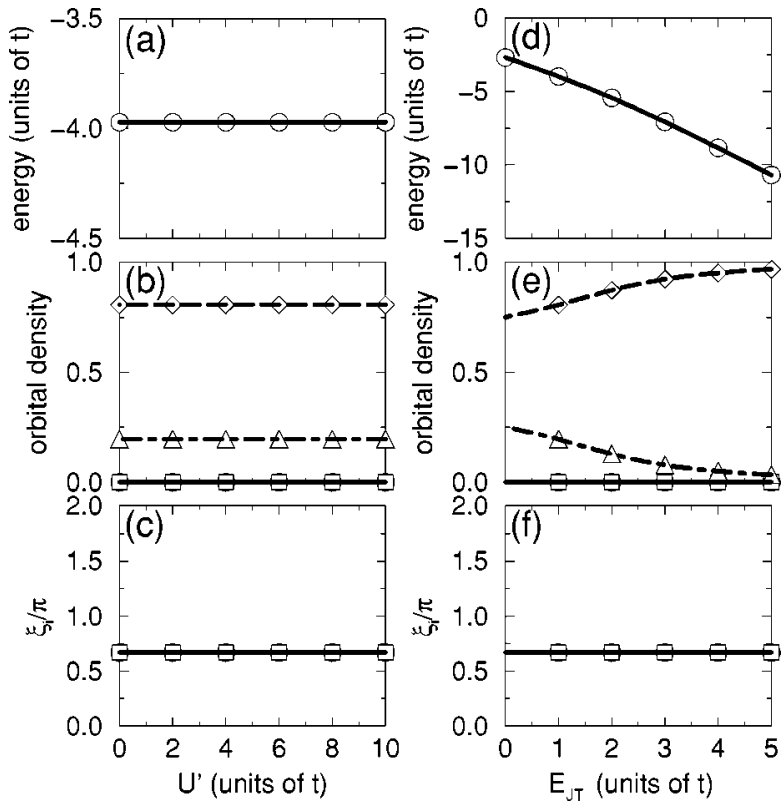

FIG. 3. Mean-field and exact-diagonalization results at $n=0.5$ for a four-site $1 \mathrm{D}$ chain with the realistic hopping amplitude along the $x$ axis. The meaning of curves and symbols are the same as those in Fig. 2.

abruptly changes due to the inclusion of the JT distortion. However, the on-site correlation is still important to achieve effectively the strong-coupling region, since $U^{\prime}$ is renormalized into the JT energy.

To check whether the statements in the previous paragraph are correct also in the doped case, the same analysis is carried out for $n=0.5$, as shown in Figs. 3(a)-3(f). Note again that the MFA results are always exact and the physical quantities are continuous as a function of $U^{\prime}$ for nonzero values of $E_{\mathrm{JT}}$, but the local orbital set cannot be uniquely determined for $E_{\mathrm{JT}}=0$. In addition, the results are independent of $U^{\prime}$ in Figs. 3(a)-3(c). This is understood as follows: If a unitary transformation is introduced to make the hopping matrix diagonal, the model $H_{\mathrm{C}}^{\infty}$ in the FM phase is reduced to

$$
H_{\mathrm{FK}}=-\sum_{\mathbf{i} \mathbf{a}}(4 t / 3) a_{\mathbf{i}}^{\dagger} a_{\mathbf{i}+\mathbf{a}}+U^{\prime} \sum_{\mathbf{i}} a_{\mathbf{i}}^{\dagger} a_{\mathbf{i}} b_{\mathbf{i}}^{\dagger} b_{\mathbf{i}},
$$

where $a_{\mathbf{i}}$ and $b_{\mathbf{i}}$ are given by $a_{\mathbf{i}}=-(\sqrt{3} / 2) c_{\mathbf{i} a}+(1 / 2) c_{\mathbf{i} b}$ and $b_{\mathbf{i}}=(1 / 2) c_{\mathbf{i} a}+(\sqrt{3} / 2) c_{\mathbf{i} b}$, respectively. In this reduced model, only the a electrons can hop and they interact with the localized b electrons, which is just the Falicov-Kimball model. ${ }^{46}$ Of course, this model is obtained trivially if the $1 \mathrm{D}$ chain along the $z$ direction is considered. Thus, the ground state at $n=0.5$ is obtained by filling the lower a band up to $n=0.5$ and $U^{\prime}$ does not work at all. When the electronphonon coupling is switched on, the system becomes insulating, but the effect of $U^{\prime}$ is still inactive. Although the perfect agreement of the mean-field results with the exact numbers is due to the special properties of the $1 \mathrm{D}$ chain, the JT-phononic model with the realistic hopping appears to contain the important physics of the problem and the electron correlation is not as crucially important as naively expected.

As for the orbital densities at $n=0.5,\left\langle\tilde{n}_{\mathbf{i a}}\right\rangle$ is always negligible small, but $\left\langle\tilde{n}_{\mathbf{i b}}\right\rangle$ for the even sites is almost unity, while $\left\langle\tilde{n}_{\mathbf{i b}}\right\rangle$ for the odd sites is small, clearly indicating the $\mathrm{CO}$ tendency. In this case, $\xi_{\mathbf{i}}$ is always given by $2 \pi / 3$ irrespective of the site position, denoting the occurrence of the ferro ordering of the $d_{3 x^{2}-r^{2}}$ orbitals. This is easily understood by the double exchange mechanism in the orbital degree of freedom. Namely, the orbital arranges uniformly to improve the kinetic energy of the $e_{\mathrm{g}}$ electrons, just as the spin does in the FM phase of the doped one-orbital model.

\section{CHARGE-ORBITAL ORDERING IN HALF-DOPED MANGANITES}

In the previous section, the $\mathrm{CO}$ state with a uniform orbital arrangement has been suggested as the ground-state of the $1 \mathrm{D}$ chain at $n=0.5$. However, in the real materials, there occurs a more complicated $\mathrm{CO} / \mathrm{OO}$ arrangement due to the variety of magnetic structures such as FM, A-type AFM, $C$-type AFM, and $G$-type AFM phase [see Fig. 4(a)]. In particular, in the narrow-band half-doped manganite such as $\mathrm{La}_{0.5} \mathrm{Ca}_{0.5} \mathrm{MnO}_{3}$ and $\mathrm{Nd}_{0.5} \mathrm{Sr}_{0.5} \mathrm{MnO}_{3}$, the so-called CE-type AFM phase is stabilized, as shown in Fig. 4(a). Note that the spin pattern in the $x-y$ plane is shown and spin directions become antiparallel along the $z$ axis. An important feature of this structure is a zigzag 1D path formed by FM array of $t_{2 \mathrm{~g}}$. As schematically shown in the right panel in Fig. 4(b), the $\mathrm{CO}$ state appears with the checkerboard pattern and the $\left(3 x^{2}-r^{2}\right) /\left(3 y^{2}-r^{2}\right)$-type orbital ordering is concomitant to this CO state. The origin of this complex spin-charge-orbital structure has been recently clarified on the basis of the topology of the zigzag structure. ${ }^{20}$ Along the $z$ axis, the CE pattern stacks with the same $\mathrm{CO} / \mathrm{OO}$ structure, but the coupling of $t_{2 \mathrm{~g}}$ spins along the $z$ axis is antiferromagnetic. If only the bipartite charge structure in the $x-y$ plane is considered, naively the nearest-neighbor Coulomb interaction $V$ seems to be the key issue in the formation of the $\mathrm{CO} / \mathrm{OO}$ phase. $^{41}$ However, if $V$ is strong enough to bring the $\mathrm{CO}$ structure in the $x-y$ plane, the 3D Wigner-crystal structure should appear in the cubic lattice, but this is not observed in the real system. Thus, $V$ is not the only ingredient needed for the stabilization of the $\mathrm{CO}$ structure of half-doped manganites, as already discussed in the Introduction. What other terms in the Hamiltonian may create a charge-stacked phase?

In order to clarify this point, in this subsection $4 \times 4 \times 4$ lattices with the periodic boundary condition are studied as a simple representation of half-doped manganites on the basis of $H^{\infty}$. In Fig. 4(c), energies for several magnetic phases are plotted as a function of $J_{\mathrm{AF}}$ for $\lambda=1.6 .^{22}$ At this stage in the calculations, $V$ is set to zero and its effect will be discussed later. The results of Fig. 4(c) are obtained from the JTphononic model, but as mentioned in the previous section, it can be interpreted that the effect of $U^{\prime}$ is included effectively in the electron-phonon coupling. Although the effect of $U^{\prime}$ also appears through the nontrivial term $\left(\widetilde{U}^{\prime} / 2\right) \Sigma_{\mathbf{i}}\left\langle n_{\mathbf{i}}\right\rangle n_{\mathbf{i}}$, this term essentially indicates the prohibition of double occupancy, and does not lead to qualitative changes in the results obtained from the JT-phononic model, as long as $\beta$ is larger than unity and $\widetilde{U}^{\prime}$ is positive. Thus, the results below can be interpreted as arising from a purely JT calculation or a mixture of JT and Coulomb interactions within a mean-field technique. The curves shown are the results in the MFA 
(a)
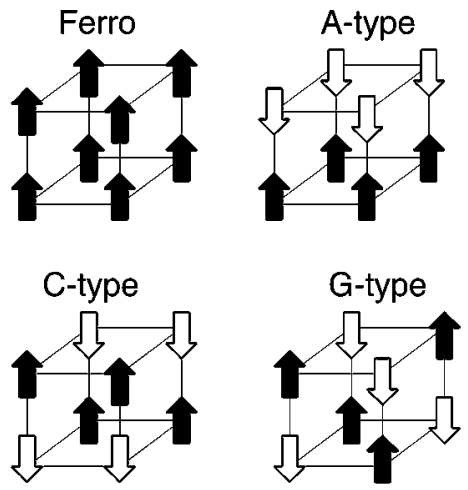

(b)
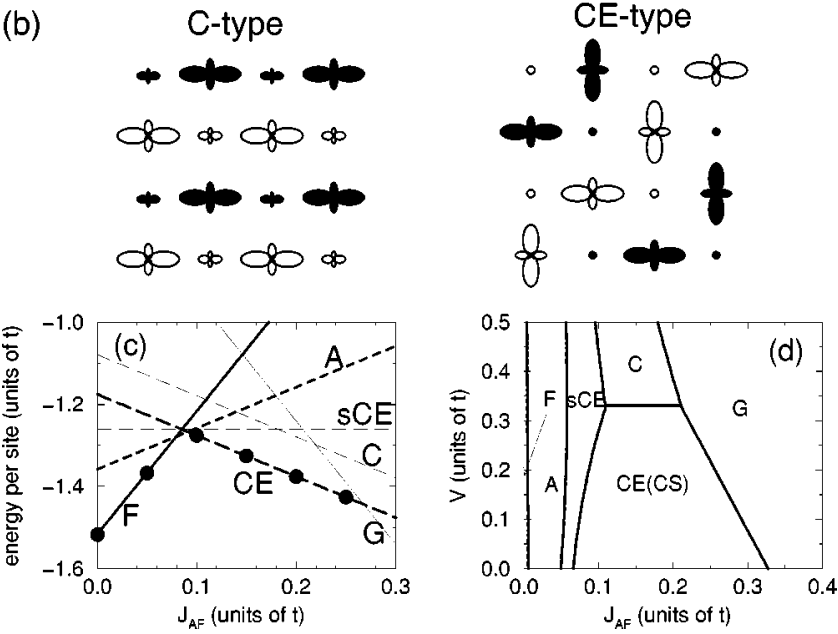

FIG. 4. (a) Spin arrangements for FM, $A$-type AFM, $C$-type AFM, $G$-type AFM, and CE-type AFM phases. Solid and open arrows indicate up and down spin, respectively. For FM, A-type AFM, $C$-type AFM, and $G$-type AFM phases, $2 \times 2 \times 2$ clusters are shown, but the structures are periodically repeated in all directions. For CE-type AFM phase, the spin arrangement only in the $x-y$ plane is shown. Along the $z$ axis, this pattern is stacked, but spin directions become antiparallel. (b) Schematic representations of the spin, charge, and orbital ordering for $C$-type (left panel) and CEtype (right panel) AFM structures. Solid and open symbols indicate up and down $t_{2 \mathrm{~g}}$ spins, respectively. The lobes indicates $\left(3 x^{2}\right.$ $\left.-r^{2}\right)$ or $\left(3 y^{2}-r^{2}\right)$ orbitals and the change of its size symbolically indicates the spatial modulation of orbital density. The circles denote $\mathrm{Mn}^{4+}$ sites. (c) Energy per site as a function of $J_{\mathrm{AF}}$ for $\lambda$ $=1.6$ and $U^{\prime}=0$. The curves denote the mean-field results and the solid symbols indicate the energy obtained by the relaxation method. Thick solid, thick broken, thin broken, thick dashed, thin dashed, thin broken, and thin solid lines denotes FM, $A$-type, shifted CE-type, CE-type, $C$-type, and $G$-type states, respectively. (d) The phase diagram in the $\left(J_{\mathrm{AF}}, V\right)$ plane. Note that the charge-stacked structure along the $z$ axis can be observed only in the CE-type AFM phase.

obtained for several fixed $t_{2 \mathrm{~g}}$ spin patterns, while the solid circles are obtained by the optimization of both the local JT distortion and the $t_{2 \mathrm{~g}}$ spin angles simultaneously. The agreement between the analytic and numerical results is excellent, indicating that the MFA works quite well in the JT-phononic model. For $J_{\mathrm{AF}} \leq 0.1 t$, a metallic 3D FM phase is stabilized, since this spin pattern optimizes the kinetic energy. In a very narrow region around $J_{\mathrm{AF}} \approx 0.1 t$, the $A$-type $\mathrm{AFM}$ phase occurs. This phase is metallic in the present intermediate cou- pling, but the uniform $\left(x^{2}-y^{2}\right)$-type orbital ordering appears. In the wider region $0.1 t \leq J_{\mathrm{AF}} \leq 0.25 t$, the CE-type AFM structure is the ground state. For unrealistic large values of $J_{\mathrm{AF}}$, the $G$-type AFM phase is stabilized to gain the magnetic energy.

Now let us focus our attention on the stabilization of the CE-type phase. Due to the competition between the kinetic energy of the $e_{\mathrm{g}}$ electrons and the magnetic energy of the $t_{2 \mathrm{~g}}$ spins, the $1 \mathrm{D}$ stripe-like AFM configuration occurs, ${ }^{20}$ in which arrays of $t_{2 \mathrm{~g}}$ spins order ferromagnetically along some particular 1D paths. Precisely the shape of the 1D FM path, the zigzag path, is a key to understanding the CE-type phase. To see this, let us compare the CE state with the $C$-type AFM state, which is characterized by the straight-line FM path, as shown in the left panel in Fig. 4(b). Although the energy of the $C$-type structure has the same slope as a function of $J_{\mathrm{AF}}$ as the CE type, it is not the ground state, as shown in Fig. 4(c). As for the orbital arrangement, the $\left(3 x^{2}-r^{2}\right)$-type $\mathrm{OO}$ occurs in the $C$-type, while the $\left(3 x^{2}\right.$ $\left.-r^{2}\right) /\left(3 y^{2}-r^{2}\right)$-type orbital arrangement appears in the CEtype state, as shown in Fig. 4(b). At a first glance, these two $\mathrm{OO}$ states seem to be quite different, but if the concept of the orbital double exchange mechanism is employed, there is no essential distinction between them. Namely, in both cases, the $e_{\mathrm{g}}$ electron orbital is always polarized along the hopping direction of the 1D paths.

An essential point for the stabilization of the chargestacked CE-type AFM phase is the difference of the topology of the 1D paths between the straight and zigzag shapes. Mathematically, the topology of the 1D path can be characterized by "the winding number" $w$ (Ref. 19) defined from the Berry phase connection of the $e_{\mathrm{g}}$-electron wave function along the hopping path. ${ }^{47}$ As shown in Ref. 20, $w$ is decomposed into two terms as $w=w_{\mathrm{g}}+w_{\mathrm{t}}$. The former, $w_{\mathrm{g}}$, is called the geometric term, ${ }^{48}$ which is 0 (1) corresponding to the ferro- (antiferro-) arrangement in the orbital sector along the 1D path. As discussed above, due to the orbital double exchange mechanism, $w_{\mathrm{g}}$ is zero in the doped case irrespective of the shape of the hopping path. The difference between the straight and zigzag paths appears in $w_{\mathrm{t}}$, expressed as $w_{\mathrm{t}}=N_{\mathrm{v}} / 2$, where $N_{\mathrm{v}}$ is the number of vertices appearing in the unit of the 1D chain. ${ }^{20}$ Since $w_{\mathrm{t}}$ is determined only by the shape of the 1D path, it is called the topological term. Of course, $N_{\mathrm{v}}$ is zero for the straight path, while $N_{\mathrm{v}}$ is equal to 2 for the zigzag path. Thus, it is obtained that $w=0$ and 1 are the values for the $C$ - and CE-type AFM phases, respectively.

To understand why the zigzag chain with $w=1$ has the lower energy, it is useful to consider the situation $U^{\prime}=E_{\mathrm{JT}}$ $=0$, in which the CE-type AFM spin structure characterized by the zigzag 1D chain is stabilized in the picture of a band insulator. ${ }^{20,52,53}$ Due to the periodic change along the zigzag path in the hopping amplitude as $\left\{\cdots, t_{\gamma \gamma^{\prime}}^{\mathbf{x}}, t_{\gamma \gamma^{\prime}}^{\mathbf{x}}, t_{\gamma \gamma^{\prime}}^{\mathbf{y}}, t_{\gamma \gamma^{\prime}}^{\mathbf{y}}\right.$, $\cdots\}$, the system becomes the band insulator. Although the difference in the hopping amplitudes in the $x$ and $y$ directions is only a phase factor in $t_{\mathrm{ab}}^{\mathrm{y}}$ and $t_{\mathrm{ab}}^{\mathrm{x}}$, a bandgap of the order of $t$ develops. On the other hand, note that the $C$-type AFM states characterized by the straight path is metallic, since there is no change in the hopping amplitude from bond to bond. In Ref. 20, it has been clearly shown that the CE-type 
structure has the largest bandgap among all the possible types of zigzag hopping paths at $n=0.5$. Moreover, in Ref. 20 , if the JT energy is switched on in this phase, it has been shown that the CE-type phase continues to be the ground state and charge-ordering appears with the $\left(3 x^{2}-r^{2}\right) /\left(3 y^{2}\right.$ $-r^{2}$ )-type orbital-ordering. Thus, based on the bandinsulator picture and the spirit of the adiabatic continuation, the CE-type AFM structure characterized by the 1D zigzag FM chain is the ground state.

Now the physical meaning of the energy difference between CE- and $C$-type states is discussed using the concept of the winding number. For this purpose, an analogy with a typical spin problem is quite useful. ${ }^{48}$ In the spin problem, by classifying the states with the total spin $S$ which is a conserved quantity, the exchange energy is defined by the difference between the energies of the singlet $(S=0)$ and triplet $(S=1)$ states. In the present problem, $w$ is the topological entity and the conserved quantity. Thus, the energy difference between the states with $w=1$ and $w=0, J_{\mathrm{w}}$, is expected to play a similar role as the exchange energy in the spin problem. As for the magnitude of $J_{\mathrm{w}}$, it can be of the order of $0.1 t$ from the analysis of the two-site problem, ${ }^{48}$ although it depends on the value of $E_{\mathrm{JT}}$.

Another phase competing with the CE-type state is the shifted CE-type (sCE) structure. ${ }^{21,22}$ This is also obtained by the stacking of the 2D arrangement shown in the right panel in Fig. 4(b) along the $z$ axis, but one-lattice spacing shifted in the $x$ (or $y$ ) direction. Due to this shift, the number of FM and AFM bonds becomes equal, and the magnetic energy is exactly canceled. In fact, the energy for the $\mathrm{SCE}$ structure is independent of $J_{\mathrm{AF}}$. The CE-type phase is stabilized against the sCE-structure by the magnetic energy, as observed in Fig. 4(c), showing the key role played by $J_{A F}$ in models for manganites and likely in the real compounds as well.

Let us consider now the effect of $V$ on the CO states. For this purpose, the mean-field calculation is carried out based on $H_{\mathrm{MF}}^{\infty}$ for a reasonable parameter set ${ }^{49}$ such as $t=0.5 \mathrm{eV},{ }^{30} E_{\mathrm{JT}}=0.25 \mathrm{eV},{ }^{50}$ and $U^{\prime}=5 \mathrm{eV} .^{25}$ The phase diagram in the $\left(J_{\mathrm{AF}}, V\right)$ plane is shown in Fig. 4(d). From this figure it is clear that the charge-stacked structure occurs for the CE-type AFM phase in a broad region of parameter space, as deduced from the Fourier transform of the charge correlation which has an observed peak at $(\pi, \pi, 0)$ in the CE-type state, while a peak appears at $(\pi, \pi, \pi)$ in the sCE and $C$-type AFM states. ${ }^{21}$ A remarkable fact of Fig. 4(d) is that the charge-stacked structure is robust against the inclusion of $V$. The boundary between the SCE and CE phases is qualitatively understood by the balance of the magnetic energy gain and the charge repulsion loss. ${ }^{22}$ On the other hand, the phase boundary between the CE- and $C$-type AFM states is independent of $J_{\mathrm{AF}}$, since those two states have the same magnetic energy. As mentioned above, in this case, the energy $J_{\mathrm{w}}$ due to the difference in the topology of 1D path stabilizes the charge-stacked structure in spite of the charge repulsion loss. In fact, the phase boundary exists around $V$ $\approx 0.3 t$, which is the same order as $J_{\mathrm{w}}$. Although it is difficult to know the exact value of $V$ in the actual material, if the simple screened Coulomb interaction is estimated using the large dielectric constant of manganites, ${ }^{51} \mathrm{~V} / \mathrm{t}$ is estimated to be $0.1 \sim 0.2,{ }^{11}$ i.e., inside the charge-stacked region in our phase diagram.
Finally, a comment on the stabilization of the CE-type structure in the purely Coulombic model is provided. As discussed above, in the case of $U^{\prime}=E_{\mathrm{JT}}=0$, the CE-type AFM spin structure is found to be the ground state in the bandinsulator picture. If $U^{\prime}$ is smoothly switched-on in this phase, still keeping $E_{\mathrm{JT}}=0$, the CE-type phase still continues to be the ground state and charge-ordering appears even without the help of $V$, since the local charge in the straight segment of the zigzag chain is larger than that at the corner site. ${ }^{53}$ The ground-state energy for the zigzag chain is lower than that for the straight chain, and its difference is again of the order of $0.1 t$. Since this energy difference can compensate the energy loss due to $V$ in the charge-stacked structure, it would be possible to understand the charge-stacked structure even in the purely Coulombic model on the same topological argument as carried out for the JT-phononic model. Thus, it is possible to fill the 3D cubic lattice by the zigzag 1D chains stacked in the $b$ - and $c$-axis directions, with the same charge ordering but antiparallel $t_{2 g}$-spin directions across those 1D chains. Note, however, that the energy is invariant for the choice of local $e_{\mathrm{g}}$-electron orbital and the $\left(3 x^{2}-r^{2}\right) /\left(3 y^{2}-r^{2}\right)$-type orbital arrangement cannot be specified in the purely Coulombic model. On the other hand, in the pure JT-phononic model, the CE-type AFM spin structure, the charge-stacked $\mathrm{CO}$ state, and the $\left(3 x^{2}-r^{2}\right) /\left(3 y^{2}\right.$ $-r^{2}$ )-type OO-phase have been fully understood, as explained before in this section. Thus, based on these results, these authors believe that the purely JT-phononic model is more effective than the purely Coulombic model for the theoretical investigation of manganites, although certainly both lead to very similar physics.

\section{PHASE-SEPARATION TENDENCY}

In previous investigations using the unbiased Monte Carlo simulations, the PS tendency has been clearly established in manganite models. ${ }^{7-9,11}$ In the simulations, PS appeared both in the one-orbital FM Kondo model ${ }^{7-9}$ and the two-orbital JT-phononic models. ${ }^{11}$ PS occurs due to the balance between the kinetic energy of $e_{\mathrm{g}}$ electrons and the potential energy from the background, either the $t_{2 \mathrm{~g}}$ spins or the JT distortion. Due to this difference in the origin of the background potential, two-types of PS exist, spin driven and orbital driven. In the following, the 1D case is used for simplicity. Although the situation will be more complicated in higher dimensions, the essential physics is expected to be captured in the 1D case.

One type of PS appears in the two-orbital model driven by the spin sector between the spin FM and AFM phases, mainly in the region between $x \approx 0.5$ and $x=1.0$. To improve the kinetic energy, in this doped situation the $t_{2 \mathrm{~g}}$ spins and $e_{\mathrm{g}}$-electron orbital tend to array in a ferromanner, but in the heavily doped region close to $x=1.0$, the $t_{2 \mathrm{~g}}$ spins order antiferromagnetically to gain the magnetic energy which dominates over the kinetic energy. Thus, in this case, the PS between FM/OF and AFM/OF states appears, as clearly indicated in the previous Monte Carlo simulations. Note that the acronym in front of the slash indicates the spin state, while the acronym after the slash denotes the orbital arrangement, e.g., FM/OF indicates the spin FM and orbital ferro state. Note that this PS is possible in the one-orbital model as 
well, since the orbital degrees of freedom is not active for this case.

Another variety of PS is related to the orbital degrees of freedom coupled to the JT distortion, which mainly exists between $x=0$ and $x \approx 0.5$ in the two-orbitals model. In the undoped situation and for reasonable values of $J_{\mathrm{AF}}$, the FM/ OAF-state is the ground state, as shown in the previous section. Also in the unbiased Monte Carlo simulation, this phase has been obtained. ${ }^{11}$ When holes are doped, the spin structure is still FM, but the orbital arrangement becomes uniform to gain the kinetic energy by polarizing the orbitals along the hopping direction (orbital double-exchange mechanism). Thus, in this case, the PS between FM/OAF and FM/OF states appears, as suggested in the previous works for the two-orbital model. ${ }^{11}$

In the present MFA, the electron number $n$ has been fixed. ${ }^{54}$ For a given $n$, the optimized structure both for the $t_{2 \mathrm{~g}}$ spins and the lattice distortion are determined by the analytic MFA and the numerical technique. To understand the PS tendency in this formalism, it is necessary to check the stability of the obtained phase by calculating the ground-state energy $E_{0}$ as a function of $n$. Namely, if a negative curvature is obtained, i.e., $\partial^{2} E_{0} / \partial n^{2}<0$, such a phase is unstable even if it is the ground state at a fixed $n$. If the PS tendency is included in the present model, the FM/OF states between $x$ $=0$ and $x=0.5$ should be unstable and the mixed phase should have lower energy than the states obtained by the MFA, in order to reproduce the Monte Carlo results. In order to verify this, the energy difference $\Delta E(x)$ is plotted as a function of $x$ for $E_{\mathrm{JT}}=1.2$ in a 16-site spin-FM 1D chain with the realistic hopping along the $x$ axis. Here $\Delta E(x)$ $=E_{0}(x)-\epsilon x$ with $\epsilon=2\left[E_{0}(x=0)-E_{0}(x=0.5)\right]$. If $\Delta E(x)$ is positive, the homogeneous state is unstable and, instead, the mixed-phase appears. Note that the state at $x=0.5$ is stable. The result is shown in Fig. 5(a), in which positive $\Delta E(x)$ is indeed observed between $x=0$ and $x=0.5$. In Fig. $5(\mathrm{~b})$, the orbital arrangements at $x=0$ and $x=0.5$ are shown by depicting the shape of the occupied b orbital in the $x-y$ plane. It should be noted that the size of the orbital is proportional to $\left\langle\tilde{n}_{b \mathbf{i}}\right\rangle$. This result agrees very well with the previous Monte Carlo calculation, and the PS tendency is believed to be definitely established in the JT-phononic model.

Now let us analyze whether it is possible to detect the PS tendency in the purely Coulombic model or not. For this purpose, $\Delta E(x)$ is evaluated using a 16-site spin-FM 1D chain with the realistic hopping along the $x$ axis for $E_{\mathrm{JT}}=0$ and $U^{\prime}=10 t$ by using the mean-field Hamiltonian Eq. (18). As shown in Fig. 5(c), again the positive $\Delta E$ for $x$ between 0 and 0.5 can be observed, indicating clearly the PS tendency. If the present mean-field Hamiltonian is accepted, this result is quite natural, since $U^{\prime}$ is included effectively in the coupling between the $e_{\mathrm{g}}$ electrons and JT distortion. However, one may consider that this is just an artifact due to the MFA. Thus, in the following section, it is explicitly shown that this PS tendency in the purely Coulombic model is not due to particular properties of the MFA by performing the DMRG calculation in the 1D Hubbard-like model.

In short, it is quite interesting to observe that the MFA can properly reproduce the PS tendencies found using other more sophisticated techniques. Although the calculation is

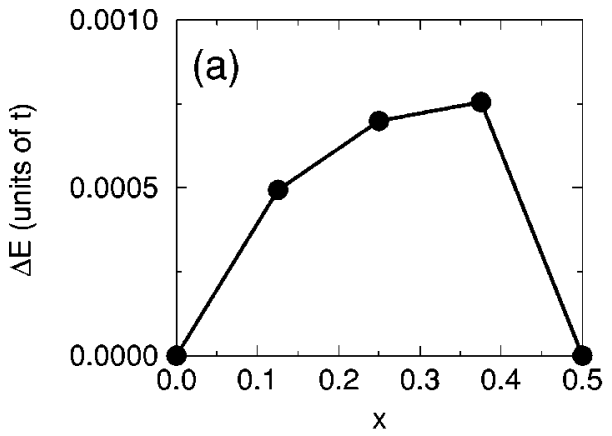

(b)
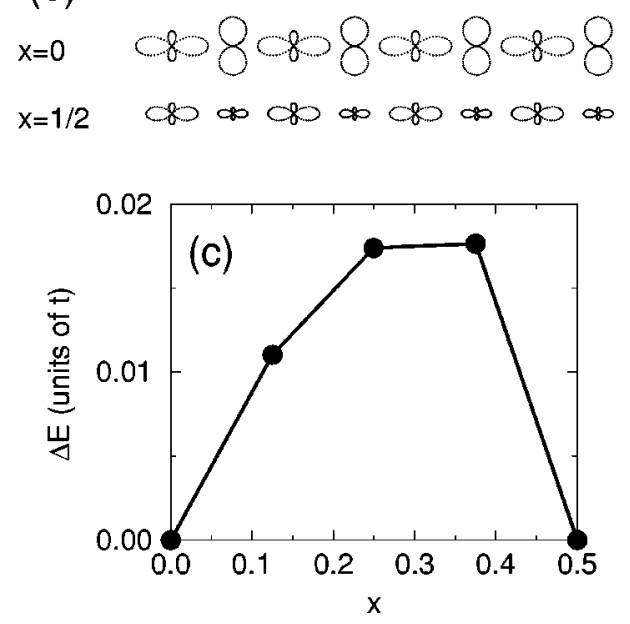

FIG. 5. (a) The energy difference $\Delta E$ as a function of $x$ for a 16-site $1 \mathrm{D}$ chain with $U^{\prime}=0$ and $E_{\mathrm{JT}}=1.2$. The definition of $\Delta E$ is shown in the text. (b) Orbital density $\left\langle\tilde{n}_{\mathrm{ib}}\right\rangle$ for $x=0$ (upper chain) and 0.5 (lower chain). Note that the $\mathrm{b}$ orbital in the $x-y$ plane is depicted and its size is proportional to $\left\langle\tilde{n}_{\mathrm{ib}}\right\rangle$. (c) The energy difference $\Delta E$ as a function of $x$ for a 16 -site $1 \mathrm{D}$ chain with $U^{\prime}=10$ and $E_{\mathrm{JT}}=0$.

fairly simple and handy the obtained result is physically meaningful and reliable. More investigations on the PS tendency by using the MFA in higher dimensions would be certainly interesting since the Monte Carlo simulations become increasingly difficult as such dimension grows, but this point will be discussed in future publications.

\section{DMRG RESULT FOR $\boldsymbol{H}_{\mathrm{C}}$}

The purpose of this section is to continue the analysis of the reduced Hamiltonians of Sec. II, this time focusing on the purely Coulombic model $H_{\mathrm{C}}$. The multiorbital Hubbard model has been addressed before using a variety of approximations, ${ }^{55}$ but here care must be taken to select the appropriate technique accurate enough to search for physics similar to the results obtained with JT phonons. The use of unbiased methods is particularly important for subtle issues such as PS. For this purpose, the model $H_{\mathrm{C}}$ described in Sec. II has been studied here with the DMRG method, ${ }^{56}$ supplemented by the exact diagonalization technique, keeping the truncation errors around $10^{-6}$ by using typically 120 states on intermediate size chains with open boundary conditions. The restriction to work in the 1D system is not severe in view of the results of previous sections and those of Refs. 7-9, that showed a strong similarity between one, two, and 
three dimensions, at least regarding the rough features of the ground state. Actually, it is important to remark that the phase diagrams described in previous work by our group are mostly based on evidence coming from the short-distance correlations calculated in our study which are not expected to depend strongly on the dimensionality. In 1D systems, the existence of genuine long-range order vs slow power-law decay of correlations is a subtle issue beyond the goals of the present analysis.

In this context, the static observables studied with the DMRG method are the spin structure factor

$$
S(k)=(1 / L) \sum_{j, m}\left\langle\mathbf{s}_{j} \cdot \mathbf{s}_{m}\right\rangle e^{i(j-m) k},
$$

the charge structure factor

$$
N(k)=(1 / L) \sum_{j, m}\left\langle\rho_{j} \rho_{m}\right\rangle e^{i(j-m) k},
$$

and the orbital structure factor

$$
T^{z}(k)=(1 / L) \sum_{j, m}\left\langle T_{j}^{z} T_{m}^{z}\right\rangle e^{i(j-m) k}
$$

where $k$ is momentum, $j$ and $m$ denote site positions, $L$ is the length of the 1D chain, $T_{j}^{z}=\left(\rho_{j \mathrm{a}}-\rho_{j \mathrm{~b}}\right) / 2$, and $\rho_{j \gamma}$ $=\Sigma_{\sigma} \rho_{j \gamma \sigma}$. It is important to remark that the analysis described below has focused on the analogies with the results found using JT phonons, especially regarding PS and the existence and properties of the spin-AF orbital-staggered phase at density $n=1$. In the several other interesting phases reported in this section, our effort has been limited to the description of their main features regarding their spin, charge, and orbital characteristics, postponing for a future publication a more detailed analysis of its origin and possible relevance to experiments. In order to focus on the similarity in the effects of the on-site correlation and the JT phonons, $V$ is set to be zero for the time being. The influence of $V$ will be discussed later. Note that the energy unit is $t$ in this section, as in the previous ones.

\section{A. Unit hopping matrix}

Thus far the realistic hopping matrix with nonzero offdiagonal hopping amplitude has been used. This hopping matrix is quite important to understand the properties of manganites, but the analysis is sometimes complicated due to the nonzero off-diagonal element. In order to analyze the two-orbital model more easily, it is useful to introduce first a simple unit hopping matrix for any direction, given by $t_{\gamma \gamma^{\prime}}^{\mathbf{a}}$ $=t \delta_{\gamma \gamma^{\prime}}$, where $\delta_{i j}$ is the Kronecker's delta. Besides its simplicity, this hopping amplitude has been used before in the search for the FM state in the multiorbital Hubbard model with a variety of techniques, ${ }^{55}$ and thus, it is meaningful to investigate its properties. In addition, it will be theoretically interesting to compare results using different hopping sets, to study the dependence of the ground-state properties with those amplitudes. It will actually be concluded that the use of realistic hoppings is very important in this context.
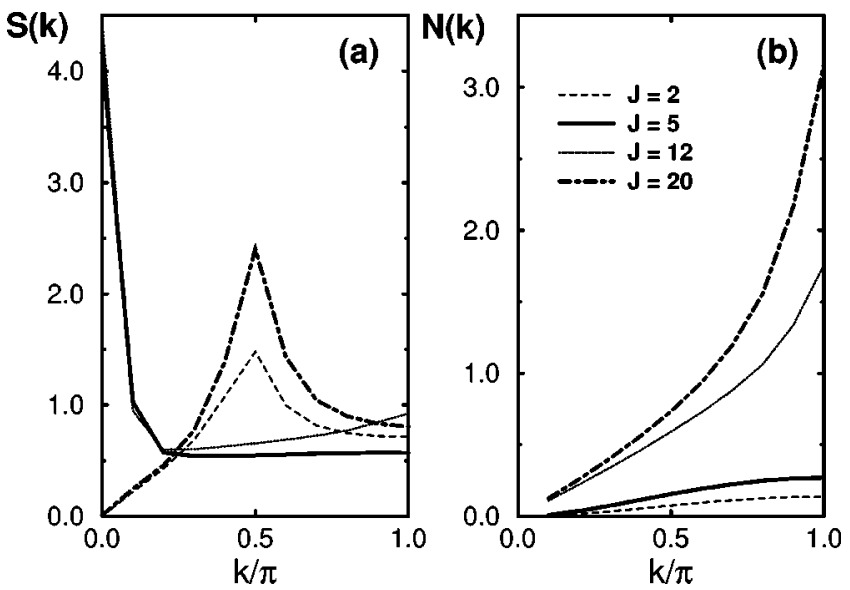

FIG. 6. DMRG results for (a) spin structure factor $S(k)$ and (b) charge structure factor $N(k)$ as a function of $k$ for $H_{\mathrm{C}}$ with the unit hopping matrix in the 20-site $1 \mathrm{D}$ chain for $U^{\prime}=10$. The dashed, solid, dotted, and dotted-dashed lines correspond to $J=2,5,12$, and 20 , respectively.

\section{Case of $n=1$}

Consider first the special case of density $n=1$. Using the DMRG and exact diagonalization methods a large set of couplings $\left(U^{\prime}, J\right)$ have been investigated, but here only the most representative results are presented as examples. In Fig. 6(a), the spin structure factor $S(k)$ is shown at fixed $U^{\prime}=10$, for particular values of $J$, and three regimes are clearly identified. At small $J$ compared with $U^{\prime}$, the spin sector has incommensurate characteristics, with a maximum at momentum $k=\pi / 2$. As $J$ grows, an abrupt transition to the FM state is observed, with $S(k)$ now peaked at zero momentum. This last regime occurs in a robust window of $J$. At $J \sim 15$, the spin sector becomes incommensurate again with a peak in $S(k)$ at $k=\pi / 2$. The charge structure factor $N(k)$ is shown in Fig. 6(b) for the same set of parameters. It is observed that at $J=2$ and 5 the charge correlations are not enhanced since only a broad peak with low-intensity appears at $k=\pi$. However, for $J=12$ and 20 indications of charge-ordering tendencies are found, since now the $k=\pi$ result is clearly enhanced, indicating a structure with an alternation between the even- and odd-sites of the chain. Note that $J=5$ and 12 have different characteristics when analyzed using $N(k)$, while they are both FM phases according to $S(k)$.

In Fig. 7(a), the orbital structure factor $T^{z}(k)$ is presented for the same set of couplings as used in Figs. 6(a) and 6(b). In this case, enhanced orbital correlations exist in the ground state at $J=2$ due to the robust values that $T^{z}(k)$ has, particularly at $k=\pi$. This is indicative of a staggered arrangement of orbitals, similar to the results discussed before in Sec. III. A qualitatively similar but even more prominent effect occurs at $J=5$. At $J=12$ and $20, T^{z}(\pi)$ is relatively small and there is no noticeable indication of enhanced orbital correlations. The transition from the low- $J$ regime, exemplified by $J=2$, to the intermediate one $(J=5)$ is abrupt, as already observed in the study of the spin structure factor.

Repeating a similar analysis for several other values of $U^{\prime}$ and $J$ allowed us to sketch a phase diagram for the twoorbital Hubbard model at density $n=1$, which is shown in Fig. 7(b). Since the complexity of the Hamiltonian does not allow us to perform a careful finite-size study to determine 

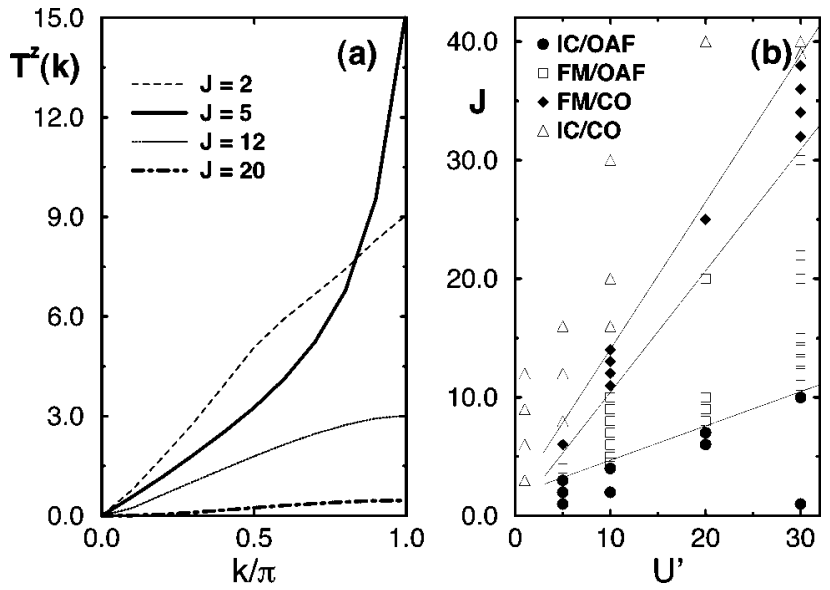

FIG. 7. (a) Orbital structure factor $T^{z}(k)$ vs $k$ using the same parameters as in Fig. 6. (b) The phase diagram of $H_{\mathrm{C}}$ for the unitmatrix hopping amplitude at $n=1$. The four phases are IC/OAF, $\mathrm{FM} / \mathrm{OAF}$, FM/CO, and IC/CO. Here 'IC', means spinincommensurate, "FM" means spin-ferromagnetic, "OAF" denotes orbital staggered, and " $\mathrm{CO}$ " indicates charge ordered.

the dominant correlations in the bulk ground state, the phase diagram of Fig. 7(b) should be considered only qualitative. Nevertheless, since for cluster sizes smaller than $L=20$ the results were found to be similar, no large size-effects are anticipated. The four identified regions are marked. Note that the emphasis has been given to the determination of the boundary of the spin-ferromagnetic orbital-ordered phase due to its similarities with the analogous phase reported in the Monte Carlo studies of the two-orbital model with JT phonons, ${ }^{11}$ and in the MFA of the previous section. The boundaries of the other regimes are less accurately determined, particularly at small values of $J$ and $U^{\prime}$.

Let us consider the origin of the many phases observed in Fig. 7. We first address the $\mathrm{CO}$ regime which appears above the line $J=U^{\prime}$. The intuitive explanation for this behavior is simple. The interorbital exchange interaction $J$ actually provides an attractive interaction to the electrons, which try to doubly populate half the sites of the chains when this interaction dominates. On the other hand, the interorbital repulsion $U^{\prime}$ prevents double occupancy of two orbitals at the same site. Then, a competition between the two occurs naturally. If $J>U^{\prime}$, which is an unphysical limit, then chargeordering occurs with roughly half the sites having two particles and the other half none. To allow for some nonzero electronic kinetic energy, the doubly occupied sites do not cluster together but are spread on the chain. The spin and charge pattern compatible with the results of Figs. 6 and 7 in the $\mathrm{CO}$ regime has a unit cell of size four lattice spacings, and an arrangement $(2 u, 0,2 d, 0)$ where $2 u$ denotes two spins up, 0 is an empty site, and $2 d$ are two spins down. Spinstaggered patterns appear frequently in the $\mathrm{CO}$ states.

The region $U^{\prime}>J$ is physically more interesting, and connected with the results observed for the model with JT phonons at the same density. The spin FM characteristic of the FM/OAF phase is believed to be caused by the optimization of the kinetic energy by spin alignment. The influence of the attractive coupling $J$ is also very important for the stabilization of this phase, since it also favors spin alignment at every site. The orbital-staggered pattern allows for some mobility of the electrons from site to site, while an orbitaluniform arrangement would not allow for that movement at density $n=1$, if all spins were aligned and when the unitmatrix hopping is used. The charge is spread uniformly, i.e., no charge-ordering exists in this phase. However, below but close to the line $J=U^{\prime}$, charge correlations are enhanced due to the proximity to the $\mathrm{CO}$ regime. In this subregion, the orbital correlations are suppressed compared with the results observed at the lower $J$ end of the FM/OAF phase, where they are maximized. Overall, it is clear that the FM/OAF state has characteristics very similar to those observed when JT phonons are used to mediate the interaction between electrons (see Sec. III). Then, this phase appears prominently both in studies with phonons and Coulombic interactions, and likely it will be stable when a mixture of the two terms is used.

As $J$ is decreased for fixed $U^{\prime}$, the ferromagnetic tendencies are naturally also reduced. The computational study shows that a regime with sharp spin incommensurate characteristics dominates in this region. The orbital order remains staggered and the charge is uniformly spread. This state is not directly related with the goals of the paper, and thus, the discussion of its origin and characteristics is postponed for future work.

\section{Case of $n \neq 1$}

One of the main goals of the study in this section is the investigation of whether PS tendencies appear in a multiorbital model having only Coulomb interactions, starting at $n$ $=1$ in the FM/OAF regime previously observed with JT phonons. For this purpose, here the couplings were fixed to $U^{\prime}=30$ and $J=22$, i.e., inside the FM/OAF phase of Fig. 7(b), and the density was varied using a cluster of 20 sites. As the number of electrons $N$ was changed between 6 and 28 (only using an even number), it was observed that the ferromagnetic characteristics persist and $S(k)$ continued to be sharply peaked at zero momentum.

Regarding charge ordering, an enhancement in this channel was found at density $n=0.5$ as can be observed in Fig. 8 (a) where results for $N=8,10,16$, and 20 are presented. This enhancement shows that at the couplings studied here tendencies toward a $\mathrm{CO}$ state are developed at $n=0.5$, in agreement with recent Monte Carlo studies for cooperative JT phonons, ${ }^{22}$ the analysis of the previous sections with noncooperative phonons, and with experiments for manganites. ${ }^{2}$ The results in the orbital sector are shown in Fig. 8(b). As the density is reduced, or increased, starting at $n=1$ the orbital correlations develop incommensurate characteristics, which is a curious effect not observed before to the best of our knowledge. When the density reaches $n=0.5, T^{z}(k)$ peaks at $k=\pi / 2$, effect likely correlated with the precursors of charge-ordering found in $N(k)$.

Note that with the DMRG and exact diagonalization techniques which are setup in the canonical ensemble where the number of particles can be fixed arbitrarily, the stability of the various states with $N$ electrons cannot be addressed directly. For this purpose it is necessary to compare the energies of the various ground states and construct a plot of the density $n$ vs $\mu$ following steps already described in detail in previous publications, ${ }^{8}$ and in the previous section. Figure 9(a) shows that all the densities studied here are actually 

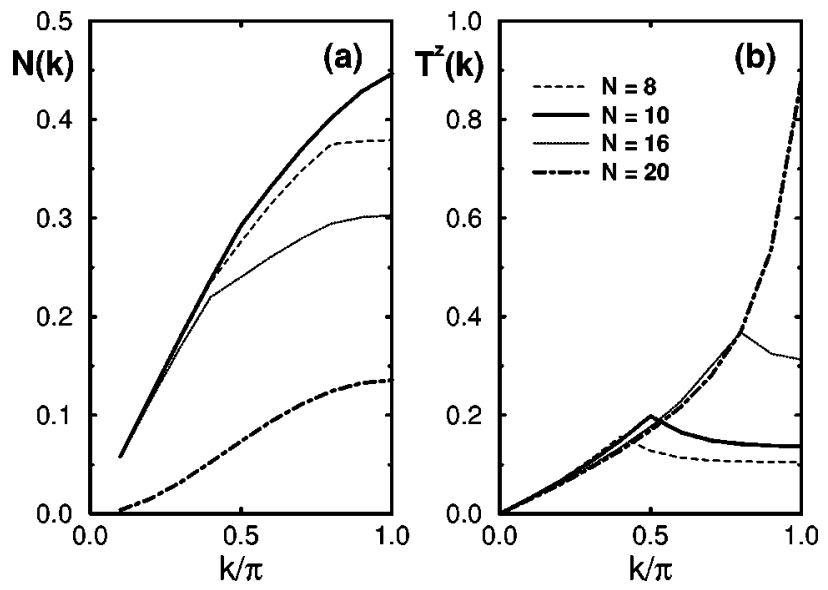

FIG. 8. (a) DMRG result for the charge structure factor $N(k)$ vs $k$ at $n=1$ for $H_{\mathrm{C}}$ with the unit matrix hopping amplitude, and a 20 -site 1D chain. $U^{\prime}$ and $J$ are fixed as 30 and 22, respectively, corresponding to couplings inside the FM/OAF phase in Fig. 7(b). The number of electrons $N$ is shown in the figure. Note that the $k$ $=\pi$ response is enhanced at density $n=0.5$, at least relatively to the other densities. (b) The orbital structure factor $T^{z}(k)$ vs $k$ using the same convention and couplings as in (a). Note the appearance of incommensurate characteristics in this channel as the density changes away from $n=1$.

stable in the sense that a finite window of $\mu$ exists for all of them where the state under study minimizes the energy. This has to be contrasted with the results found in the Monte Carlo and mean-field calculations with JT phonons where states in a finite window of $n$ were found to be unstable, ${ }^{11}$ i.e., there was no value of $\mu$ that render them the global ground state of the system. Then, it is concluded that the two-orbital Hubbard model with unit-matrix hopping studied here does not phase separate in spite of having characteristics
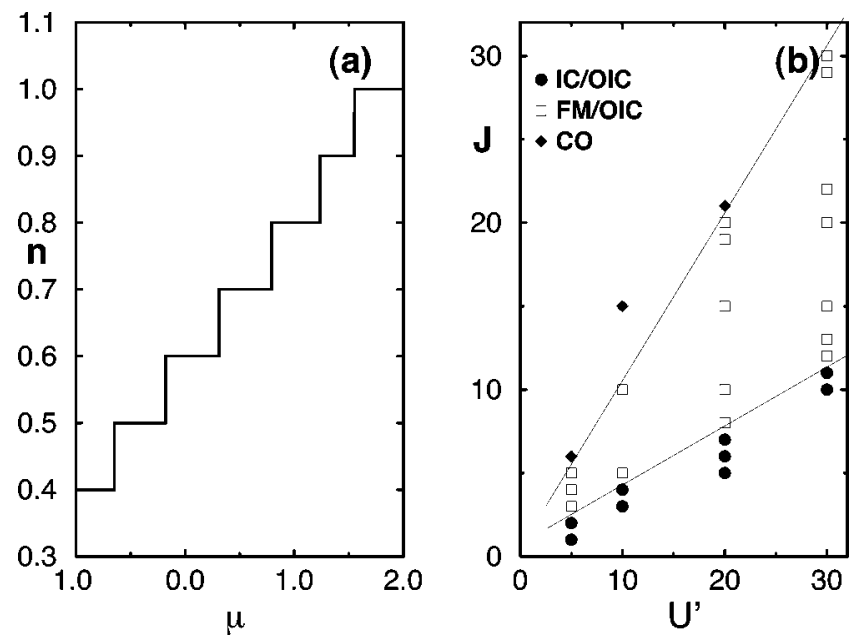

FIG. 9. (a) Density $n$ vs $\mu$ constructed from the ground-state energies corresponding to several number of electrons on a chain with 20 sites, and the couplings used in Fig. 7. Note that all densities are accessible for some values of $\mu$. (b) The phase diagram of $H_{\mathrm{C}}$ for the case of the unit hopping matrix at $n=0.5$. The two phases identified are IC/OIC and FM/OIC, where "OIC" means charge-disordered orbital incommensurate. The charge-ordered regime above $J=U^{\prime}$ has not been studied in as much detail as in Fig. $7(\mathrm{~b})$, and it is simply denoted by CO.

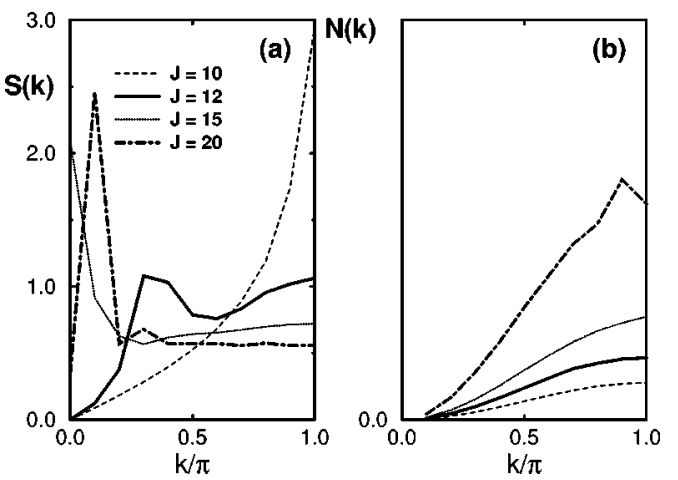

FIG. 10. (a) Spin structure factor $S(k)$ vs $k$ using the realistic hopping matrix along the $x$ axis for a 20-site 1D chain at $n=1$ and $U^{\prime}=30$. The dashed, solid, dotted, and dotted-dashed lines correspond to $J=10,12,15$, and 20 , respectively. (b) The charge structure factor $N(k)$ vs $k$ for the same parameters as in (a).

at $n=1$ very similar to those observed in the Monte Carlo simulations of the JT model at the same density. Having a FM/OAF state at $n=1$ apparently is not sufficient for PS to occur. ${ }^{57}$ This issue is conceptually important and will be addressed again in the next subsection when results with a more realistic nondiagonal hopping matrix are analyzed.

Due to the stability of the intermediate phases away from $n=1$ it is not too surprising to observe a phase diagram at $n=0.5$ with similar characteristics to those found in Fig. 7(b). In Fig. 9(b), the unphysical region $J>U^{\prime}$ has only been analyzed briefly, just sufficiently to confirm that $\mathrm{CO}$ characteristics exist there, with relevant momentum $k=\pi / 2$. In the regime $J<U^{\prime}$, the spin FM and incommensurate phases are still stable, and $T^{z}(k)$ presents a broad peak at $k=\pi / 2$ compatible with the tendencies toward charge-ordering that appear at $n=0.5$. The probable pattern of orbitals here may have orbital a below $\mathrm{b}$ at site $i$, a mostly empty $i+1$ site, the reverse orbital pattern at $i+2$, and another empty site at $i$ +3 , with this arrangement repeated in space.

\section{B. Nondiagonal hopping matrix}

The unit hopping matrix used in the previous subsection is a simple and natural choice to gather qualitative information about the two-orbital problem. However, the studies of models designed for manganites need a more complicated hopping matrix, as shown in Sec. III. In this subsection, the realistic hopping amplitudes along the $y$ direction are used to contrast the results against those obtained with the unit hopping matrix. The results are actually found to be dramatically different at densities different from unity. The organization of the subsection is similar to the previous one, i.e., the analysis starts with density $n=1$, establishing the main features of the phase diagram, and continues with $n \neq 1$ with emphasis on the possible appearance of PS.

\section{Case of $n=1$}

In Fig. 10(a), the spin structure factor $S(k)$ is shown at $U^{\prime}=30$ for representative values of $J$. At $J=10$ and smaller, the signal was found to be clearly antiferromagnetic with a sharp peak at $k=\pi$. In the intermediate region, the spin structure is complex with some incommensurate characteristics, as exemplified by the result at $J=12$. For larger values 

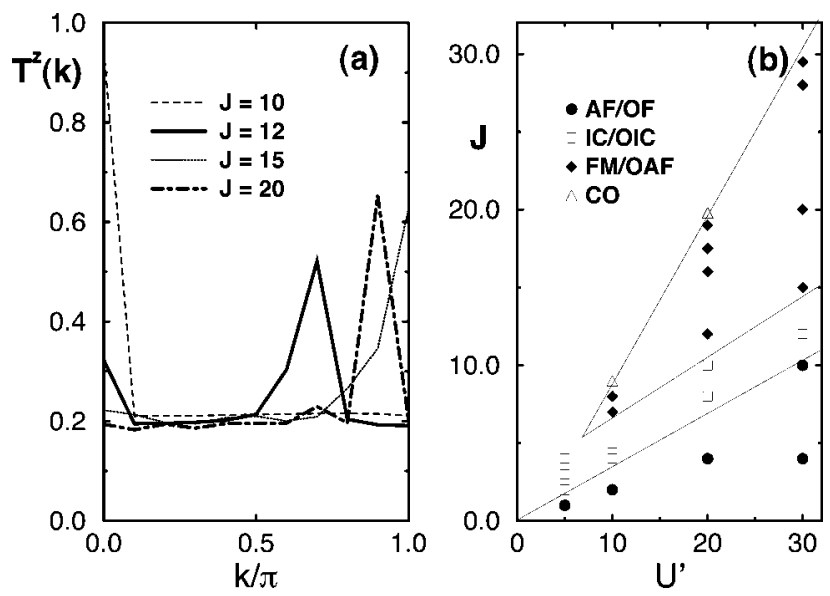

FIG. 11. (a) Orbital structure factor $T^{z}(k)$ vs $k$ for the same parameters as in Fig. 10(a). (b) The phase diagram for $n=1$ for the realistic hopping matrix. The four phases are AF/OF, IC/OIC, FM/ $\mathrm{OAF}$, and $\mathrm{CO}$, where "OF" denotes orbital-uniform and chargedisordered. The "CO" has not been characterized in detail besides its $\mathrm{CO}$ properties.

of $J$, such as 15 and 20, the system becomes ferromagnetic or quasiferromagnetic. If $J$ is increased further beyond the $J$ $=U^{\prime}$ boundary, spin incommensurate structures have been observed, as in the study leading to Fig. 7(b) (results in this unphysical regime will not be discussed further here). In Fig. $10(\mathrm{~b})$, the charge structure factor $N(k)$ is shown at the same couplings used in Fig. 10(a). This quantity only develops some nontrivial structure as the $J=U^{\prime}$ line is approached. In this case indications of charge ordering at $k=\pi$ are observed. In Fig. 11(a), the results corresponding to the orbital structure factor are shown. At small $J$, the order is uniform since $T^{z}(k)$ peaks at $k=0$. At $J=12$, an incommensurate structure appears in the orbital sector, similar to the results obtained for the unit matrix hopping away from $n=1$, and related with the spin incommensurability observed in $S(k)$. For $J=15$ and 20, a robust orbital-staggered pattern is reached.

With the information obtained at $U^{\prime}=30$ and various $J$ 's, supplemented by results gathered at $U^{\prime}=5,10$, and 20 , a rough phase diagram can be constructed, shown in Fig. 11(b). In this case four regimes are identified. At small $J$, an $\mathrm{AF} / \mathrm{OF}$ state appears which does not exist for the unit-matrix hopping. The reason is the following: For the realistic hopping matrix, orbital a has more mobility than the other. Then, to improve the kinetic energy the ground state prefers to have those orbitals as the lowest-energy ones at every site. However, at $n=1$, a spin-aligned orbital-uniform arrangement would not have any mobility due to the Pauli principle. Then, the optimal situation is achieved with antiferromagnetic order in the spin sector. The presence of this state is the main difference at $n=1$ between the results found for the unit hopping matrix shown in Fig. 7(b) and the results of Fig. 11(b). On the other hand, note that the AF/OF order does not take advantage of $J$ since the use of the hoppings in this state leads to antiparallel spins at the same site and, thus, to an energy penalization proportional to $J$. For this reason, as the coupling $J$ grows, a transition is expected to a state in close competition with the AF/OF one, namely, the FM/OAF state that has appeared several times in our investigations. In the
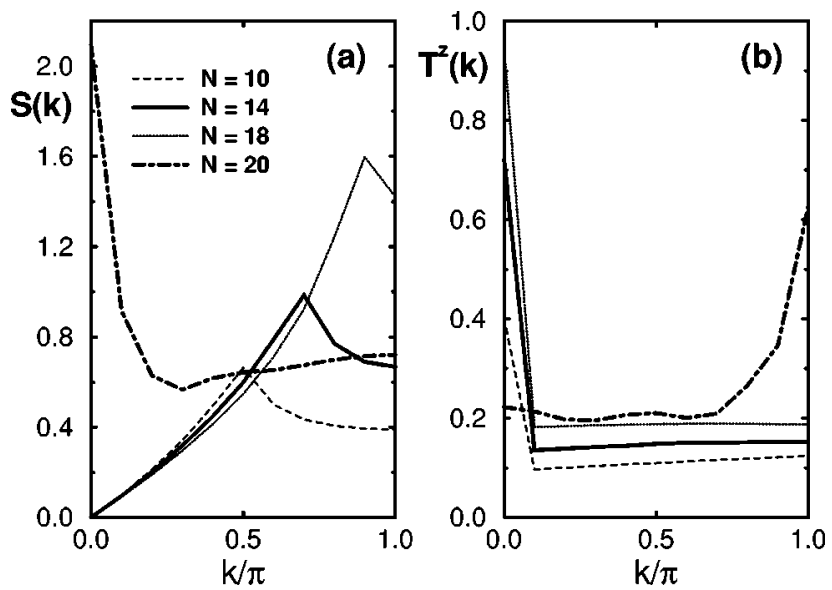

FIG. 12. (a) Spin structure factor $S(k)$ vs $k$ for the realistic hopping matrix, $U^{\prime}=30$, and $J=15$ in the 20-site chain for several electron numbers. Incommensurate characteristics appear abruptly upon doping the $n=1$ state. (b) Orbital structure factor $T^{z}(k)$ vs $k$ for the same parameters and electron number as in (a). Again an abrupt change from OAF to OF features is observed as $n$ is reduced from unity.

latter $J$ plays an important role since it favors spins alignment when two electrons visit the same site, leading to a spin ferromagnetic state. To optimize the kinetic energy, the optimal orbital pattern must be staggered, as discussed in the previous section when explaining Fig. 7(b). The interpolation between these two competing states is difficult to predict and the computational work suggests that it proceeds through a complicated mixture of FM- and AF-orbital characteristics that lead to an overall spin and orbital incommensurate pattern. Only further studies incorporating finite-size analysis will clarify if this regime is indeed stable in the bulk limit.

\section{Case of $n \neq 1$}

It is important to investigate how the phase diagram shown in Fig. 11(b) evolves as a function of density. To establish a connection with the results found in the case of the JT phonons at large $\lambda$, once again the FM/OAF state is here studied in detail since a state with similar characteristics was indeed observed at $n=1$ (Sec. III) in the mean-field studies with phonons, and the reduction of the density led to a phase-separated regime in simulations. ${ }^{11}$ Would the same occur in the purely Coulombic case with nondiagonal hopping? To gain insight, $S(k)$ is shown in Fig. 12(a) at some representative densities using couplings $U^{\prime}=30$ and $J=15$ that correspond to a point inside the FM/OAF phase of Fig. 11(b). It is interesting to observe an abrupt change in $S(k)$ when $n$ is varied from 1 to 0.9 , with an incommensurate structure appearing in the latter. This incommensuration continues up to $n=0.5$. The charge structure factor $N(k)$ also presents an abrupt change away from $n=1$, with tendencies to charge ordering maximized at $n=0.5$ (not shown). In addition, $T^{z}(k)$ drastically switches from an orbital-staggered pattern at $n=1$, to a uniform arrangement at $n \neq 1$ as shown in Fig. 12(b). At $n=0.5$ and working with $U^{\prime}=10$ and 30, it was observed that the spin-incommensurate and orbitaluniform characteristics are independent of $J$ to a good approximation, as long as $U^{\prime}>J$. 

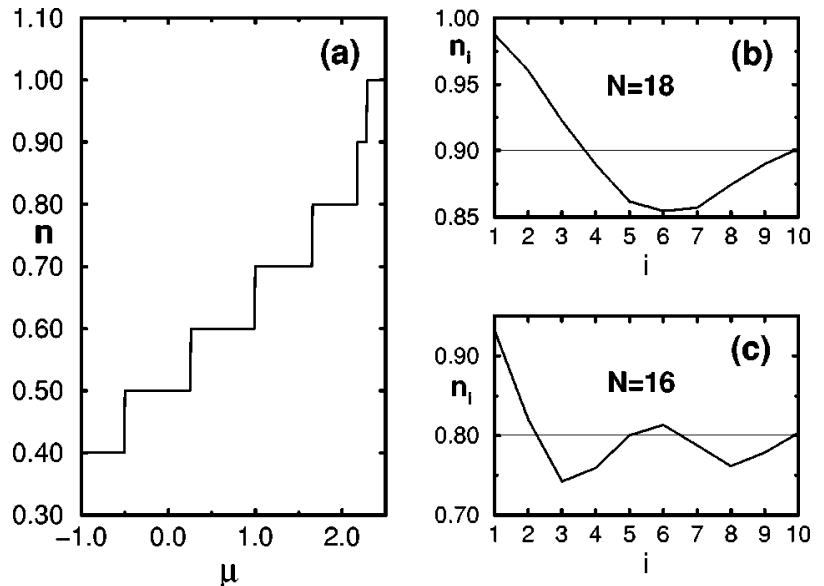

FIG. 13. (a) Density $n$ vs $\mu$ for the same parameter as in Fig. 12(a). Precursors of PS at density close to unity are observed, as discussed in the text. (b) Local density $n_{i}$ vs site position $i$ for 18 electrons on a 20-site chain. Only half the chain is shown, the results for the other half are simply obtained by reflection. The system is far from uniform in the charge sector. (c) Same as (b) but for 16 electrons.

To understand the curious behavior reported in Figs. 12(a) and 12(b), the density vs $\mu$ is plotted in Fig. 13(a) to search for the PS tendency. An anomalous behavior is observed at $n=0.9$, which has a tiny $\mu$ window of stability, and thus, a large compressibility $\kappa$ (since $\kappa$ is proportional to $d n / d \mu$ ). This behavior indicates a tendency toward PS in the data, and it may occur that small additions to the two-orbital Hubbard model such as JT phonons or even the analysis of slightly larger clusters, may render the system truly phase separated. To confirm the presence of PS precursors, the local density $n_{i}$ is shown in Fig. 13(b) at $n=0.9$. Note that the DMRG method works with open boundary conditions, and $n_{i}$ is not necessarily equal to $n$ at every site due to the lack of translational invariance. It is clear from this figure that large charge inhomogeneities are present in the ground state, while at $n=1$ (not shown) $n_{i}$ is almost uniform. Only the center of the chain has a density equal to the average one (0.9), while the ends of the chain have $n$ close to unity, and at other sites near the center the density is smaller than 0.9. The minimum in the local density shown in Fig. 13(b) may correspond to a hole doped into the $n=1$ system, that has developed polaronic characteristics. By symmetry, the other hole is on the other half of the chain. In Fig. 13(c), two holes generate two minima in the density (again, with the other two holes on the other chain half). These results show that tendencies toward charge inhomogeneities are present in this system, and precursors of PS are observed possibly in the form of polaronic behavior. The purely Coulombic model appears to be at the verge of phase separation.

To search for more clear indications of phase separation, the Coulombic interaction $U^{\prime}$ was reduced to 10 , and $J$ was fixed to 7. This still corresponds to a point at $n=1$ inside the spin-FM orbital-staggered phase. The spin and orbital structure factors for several electrons were calculated in this case, and the results (not shown) have clear similarities with those obtained at $U^{\prime}=30$. However, in this case now the analysis of the density vs $\mu$ reveals strong phase separation characteristics between densities 1.0 and 0.7 [Fig. 14(a)], qualita-
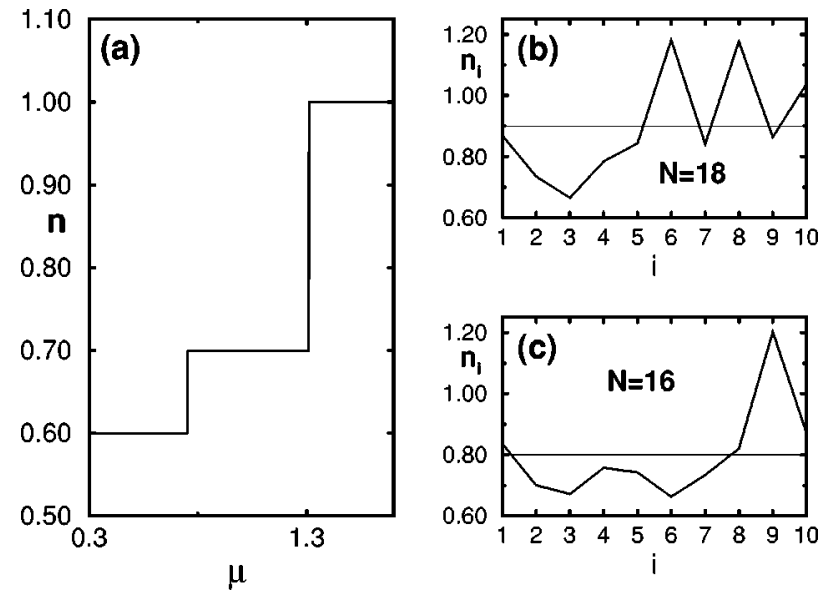

FIG. 14. (a) Density $n$ vs $\mu$ for $U^{\prime}=10$ and $J=7$. Clear phase separation between densities 1.0 and 0.7 is observed. (b) Local density $n_{i}$ vs site position $i$ for 18 electrons on a 20-site chain. Only half the chain is shown, the results for the other half are simply obtained by reflection. The system is far from uniform in the charge sector. (c) Same as (b) but for 16 electrons.

tively similar to those reported using JT phonons. In Figs. 14(b) and 14(c), the local density away from $n=1$ is shown. As in the case reported in Figs. 13(b) and 13(c), strong oscillations reveal clear tendencies to phase separation. Studies at $U^{\prime}=20$ and $J=12$ but with the realistic hopping matrix have also been carried out as part of this effort. The results are very similar to those shown in Figs. 13 and 14. Summarizing, either clear phase separation or a strong tendency to such phenomenon exists in the 1D purely Coulombic model as long as the hopping matrix is nondiagonal.

\section{Influence of nearest-neighbor repulsion}

As discussed in Sec. IV, $V$ should not be too large in the actual material, since if it were strong, the charge-stacked structure would be destroyed. However, it is important to understand whether the results change or not with the inclusion of $V$ using the same unbiased technique as in the previous subsection.

First let us consider density $n=1$, on a chain of 20 sites and $U^{\prime}=30$. In this case, few modifications were observed compared with the results obtained for $V=0$. Namely, (i) the boundary between the spin-AFM and spin-incommensurate phases at small $J$, compared with $U^{\prime}$, shifted toward a smaller $J$, and (ii) close to $J=U^{\prime}$ the charge correlations were enhanced substantially favoring the staggered pattern of charge between $n_{i}=0$ and 2, which is not penalized by $V$. Even at values such as $J=20$, i.e., not too close to $J=U^{\prime}$, this enhancement was noticeable. However, since there are no indications of such a pattern in experiments for manganites at $n=1$, there is no need to analyze this region in more detail in the present study.

At $n=0.5$, it is naively expected that the $V$ term brings the $\mathrm{CO}$ state, although only precursors of $\mathrm{CO}$ behavior were observed at $V=0$ and this density, as discussed in the previous subsection. In Fig. 15(a), $N(k)$ is shown at $U^{\prime}=10$ and $J$ $=6$ both at $V=0$ and 4 . The region near $k=\pi$ is clearly enhanced by $V$ as expected. The real-space density is shown in Fig. 15(b) and a clear charge-staggered pattern is visible. 

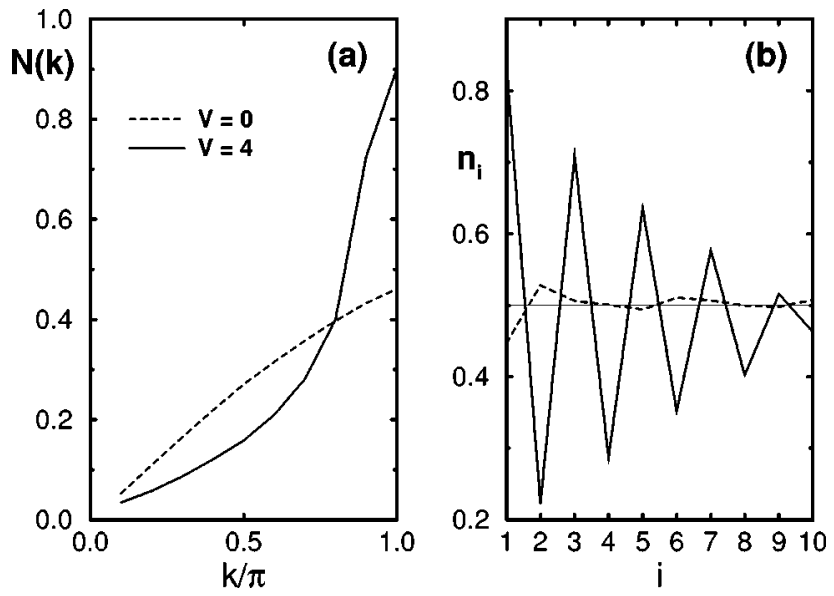

FIG. 15. (a) Charge structure factor $N(k)$ vs $k$ at $n=0.5$ with the realistic hopping for $U^{\prime}=10$ and $J=6$ in the 20-site chain. Two values of $V$ as shown and the enhancement of charge order is clearly observed. (b) The local density $n_{i}$ vs $i$ for the same parameter as in (a). Shown are results for half the lattice, the other half can be found by reflection.

The spin structure factor (not shown) is still peaked at $k$ $=\pi / 2$ as at $V=0$, compatible with a staggered spin arrangement involving the occupied (even or odd) sites. The orbital structure factor still has a large uniform component, although it has also developed a broad low-intensity peak at $k=\pi$. Overall the characteristics of the state stabilized by $V$ are orbital-uniform, charge staggered with periods of two lattice spacings, and spin staggered over the occupied sites (period of four lattice spacings). Thus, only when a sufficiently large nearest-neighbor repulsion is included, the $n=0.5 \mathrm{CO}$ state is similar to that found in the JT-phononic model reproduced in the purely Coulombic model in one dimension. This result seems in contradiction with the discussion about the chargestacked structure observed in the half-doped material, since it was concluded that $V$ is not the only origin of the $\mathrm{CO}$ state in the manganite. However, as briefly discussed in Sec. III C, if the zigzag 1D chain, not the straight $1 \mathrm{D}$ chain, is used in the calculation, the $\mathrm{CO}$ phase can be obtained even in the purely Coulombic model without $V$, although the $\left(3 x^{2}-r^{2}\right) /\left(3 y^{2}\right.$ $\left.-r^{2}\right)$-type $\mathrm{OO}$ phase cannot be obtained. From this viewpoint, a DMRG study carried out on the zigzag 1D chain will be interesting, but this is left for the future.

\section{Partial summary}

The analysis of the two-orbital model that contains only Coulomb interactions was instructive in several respects. For instance, the study has shown that a spin-ferromagnetic orbital-staggered (FM/OAF) phase appears naturally in this context at $n=1$ for a variety of hopping amplitudes, result in excellent agreement with those observed for a purely JT model, also in agreement with the previous exact diagonalization studies of Coulombic models. ${ }^{55}$ Then, the FM/OAF phase is a robust feature of models for manganites and approximations that attempt to describe these materials cannot neglect orbital ordering. Another important result of this section has been the observation of PS tendencies with Coulombic-only interactions. This was shown to occur in the form of robust precursors of this tendency in some regions of parameter space. However, to observe PS it seems necessary to use realistic electronic hopping amplitudes in the case of Coulombic models. The presence of PS tendencies both using purely JT and Coulombic interactions confirms the robustness of this feature, and it also shows that mixed-phase tendencies cannot be ignored in theoretical studies of manganites. In addition, the similarities between models with JT phonons or Coulombic repulsions suggest that the technically much simpler studies that use only phonons to mediate the interaction between electrons are qualitatively correct, and likely capture the physics of more involved models where both interactions are included.

\section{DISCUSSION AND SUMMARY}

In this paper, the two-orbital model with the JT-phononic and/or the Coulombic interactions has been studied using a variety of techniques. Three points have been confirmed in this work. (i) The main properties of manganites can be reproduced successfully by the purely JT-phononic model with a large Hund coupling even if the strong on-site correlation is not included explicitly, since the effect of such correlation can be renormalized into the effective electron-phonon coupling. (ii) In particular, in the mean-field level, the JTphononic model can successfully reproduce the CE-type AFM phase with the charge-stacked structure of the $3 \mathrm{D} \mathrm{cu}-$ bic lattice at $n=0.5$, in excellent agreement with the Monte Carlo simulations of Yunoki et al. ${ }^{22}$ Even if the nearestneighbor repulsion $V$ is introduced, this phase is not easily destroyed due to the key role played by the magnetic energy gain, regulated by $J_{\mathrm{AF}}$, in the CE- vs sCE-type competition and the "topological" energy gain in the CE- vs $C$-type competition. Particularly, it is stressed that the topology of the zigzag 1D path is the key issue leading to the stabilization of the $\mathrm{CO} / \mathrm{OO}$ state in the CE-type structure. The on-site Coulombic model treated in the mean-field approximation and without JT phonons was also found to lead to charge stacking due to the influence of $J_{\mathrm{AF}}$. (iii) The purely Coulombic model behaves in many respects very similarly to the purely JT phononic one and, in particular, it presents the phase separation tendency, especially when realistic hoppings are used.

Summarizing, both approaches to the problem of manganites, based either on Coulomb repulsions or phonons, share common tendencies. This conclusion is in agreement with the recent observation ${ }^{18}$ that the percolative character of transitions in manganites and its large magnetoresistance effect arise from the competition between metallic and insulating phases in the presence of disorder, independently of whether these phases are mainly generated by Coulombic or JT interactions. Our results have provided robust arguments suggesting that perceiving the "Coulombic" and "JT-phononic", approaches to manganites as qualitatively different ways to carry out theoretical calculations is likely incorrect.

\section{ACKNOWLEDGMENTS}

The authors thank S. Yunoki, A. Moreo, and S. Kivelson for useful conversations. T.H. has been supported by the Ministry of Education, Science, Sports, and Culture (ESSC) of Japan during his stay in the National High Magnetic Field 
Laboratory, Florida State University. T.H. was also supported by the Grant-in-Aid for Encouragement of Young Scientists under Contact No. 12740230 from the Ministry of ESSC. A.L.M. acknowledges the financial support from Fundação de Amparo à Pesquisa do Estado de São Paulo (FAPESP-Brazil). E.D. was supported by Grant No. NSFDMR-9814350.

\section{APPENDIX: RELATION WITH PHASE-SEPARATION THEORIES FOR CUPRATES}

Since a substantial portion of the paper is devoted to the issue of phase separation in models with Coulomb interactions, in this Appendix extra comments are provided regarding PS in models for high-temperature superconductors (HTSC's), issue which has been under discussion for almost a decade. ${ }^{58-60}$ In particular, it is important to clarify the relation between HTSC phase separation and the PS phenomenon discussed here for manganites. In fact, some authors strongly believe that any doped correlated insulator should have PS, and in this respect the results for manganite models would be a more particular case of a general framework. ${ }^{59}$ However, it has to be discussed in detail to what extent there is convincing theoretical evidence that indeed any doped insulator phase separates. If this is not clear, the relation between PS in cuprates and manganites weakens considerably. The discussion in this context also has to involve two other important aspects of the problem, namely the microscopic origin of PS, and its phenomenological consequences, particularly when extended Coulomb interactions are included.

In our opinion, there are several differences between the PS phenomena proposed for manganites and cuprates. (i) The properties of the two competing phases are not the same. In manganites, an undoped AFM phase and a hole-doped FM state are involved, while in cuprates it is an undoped AFM state and a hole-doped paramagnet or superconductor. In models for HTSC's, ferromagnetism does not play an active role at realistic $J / t$ couplings, while it is crucial in manganites. ${ }^{61}$ Note that the AFM state that is doped in 3D real manganites has staggered spin order only in one direction, while it is ferromagnetic in the other two ( $A$-type AFM order). In fact, PS can occur in the two-orbitals models for manganites in 1D and 2D without actually involving the AFM state, but only having two competing FM states, as discussed in the main text. ${ }^{11}$ Here the orbital degrees of freedom and JT phonons play the key role needed for PS, while they are not important in the cuprates. (ii) PS in models for manganites occur even in one dimension in regions of couplings that are realistic, as described in this paper and previous ones, unlike $t-J$ model results in the same dimension where PS only happens at large values of $J / t$, and it does not occur at all in the one-band Hubbard model. (iii) The study of PS in models analyzed in the infinite dimensional limit leads to different conclusions between cuprates and manganites, although this issue is still controversial. ${ }^{7,62}$ (iv) $\mathrm{Bi}$ stripe order has been claimed to exist in manganites. ${ }^{63}$ However, recent calculations by Hotta et al..$^{20}$ have shown that the manganite bi-stripes are better interpreted as arising from zigzag conducting chains running perpendicular to the charge stripes once electron-phonon JT-couplings are switched on. For this reason, in our opinion, it is premature to establish connections between stripes in cuprates and manganites, since the former has electronic density 0.5 while the latter has density 1.0 .

Regarding the issue of whether any doped correlated insulator produces PS, the following is our understanding of the current theoretical literature for the cuprates. The most clear manifestation of PS appears in the $t-J$ model at large $J / t$, where the tendency to form pairs of holes to minimize the number of broken AFM bonds is so intense that clusters of holes are formed instead of individual pairs. Here the attractive potential energy among hole carriers originating in the AFM background dominates over the kinetic energy, that tends to spread particles apart. As $J / t$ is reduced it has been a matter of much controversy whether the PS effect survives in the realistic small $J / t$ regime of the $t-J$ model. While exact diagonalization of small clusters, ${ }^{64}$ Monte Carlo simulations, ${ }^{65,66}$ and high-temperature expansions ${ }^{67}$ suggested that at $J / t$ of order unity the effect would disappear, other arguments ${ }^{59}$ and further computational work ${ }^{68}$ opened the possibility for PS to exist at all values of $J / t$. Very recent DMRG studies ${ }^{69}$ for ladders of increasing number of legs show that previous calculations ${ }^{68}$ may not have been sufficiently accurate and the new results suggest that PS indeed only occurs at intermediate and large values of $J / t$ in the $2 \mathrm{D}$ $t-J$ model. This same conclusion becomes more clear once extra hopping amplitudes are added to the model. In this case the substantial hole mobility induced by the extra hoppings shifts the PS regime to values of $J / t$ larger than in the pure $t$-J model case. ${ }^{70,71}$ Then, the proposal that any correlated insulator when hole doped should become phase separated is still not confirmed using unbiased techniques in simple models for cuprates.

Regarding the phenomenological aspects of the PS regime, the issue of microscopic PS that may appear in manganites has certainly been discussed before for cuprates. ${ }^{58,59}$ In this context the Coulomb interactions break into small pieces the macroscopic clusters of the two phases in competition, since they have different electronic densities. Stripe patterns emerged from calculations carried out mainly close to the atomic limit, ${ }^{58,72}$ where the attraction leading to PS plus the Coulomb repulsion are in competition. In the context of nuclear physics similar patterns have also been discussed. ${ }^{73}$ In addition, Nagaev studied the formation of finite-size clusters of one phase embedded into the other, mainly for antiferromagnetic semiconductors. ${ }^{74}$ Then, the simple picture of a stable state formed by small clusters of the competing phases, somewhat similar to the CDW pattern obtained in the 1D calculations with nearest-neighbor repulsions, is certainly common to manganites and cuprates. ${ }^{58,59}$ By analyzing the properties of a general Ginzburg-Landau free energy, the thermodynamic stability of stripe or other nonuniform phase was discussed. ${ }^{75}$

In short, the discussion presented in this Appendix suggests that the PS phenomena in models for manganites and cuprates, while sharing the general common ingredients of any PS regime, are different in origin and they must be considered separately in their study. However, certainly the last word has not been given on this interesting issue, and more work is needed to fully clarify the potential relation between cuprates and manganites. 
${ }^{1}$ S. Jin, T.H. Tiefel, M. McCormack, R.A. Fastnacht, R. Ramesh, and J.H. Chen, Science 264, 413 (1994), and references therein.

${ }^{2}$ Y. Tokura, Y. Tomioka, H. Kuwahara, A. Asamitsu, Y. Moritomo, and M. Kasai, J. Appl. Phys. 79, 5288 (1996); A.P. Ramirez, J. Phys.: Condens. Matter 9, 8171 (1997); Y. Tokura, in Colossal Magnetoresistive Oxides, edited by Y. Tokura (Gordon \& Breach, New York, 2000).

${ }^{3}$ C. Zener, Phys. Rev. 82, 403 (1951); P.W. Anderson and H. Hasegawa, ibid. 100, 675 (1955).

${ }^{4}$ P.G. de Gennes, Phys. Rev. 118, 141 (1960).

${ }^{5}$ P. Schiffer, A.P. Ramirez, W. Bao, and S.-W. Cheong, Phys. Rev. Lett. 75, 3336 (1995).

${ }^{6}$ C.H. Chen and S.-W. Cheong, Phys. Rev. Lett. 76, 4042 (1996); A.P. Ramirez, P. Scheffer, S.-W. Cheong, C.H. Chen, W. Bao, T.T.M. Palstra, P.L. Gammel, D.J. Bishop, and B. Zegarski, ibid. 76, 3188 (1996); S.-W. Cheong and C.H. Chen, in Colossal Magnetoresistance, Charge Ordering, and Related Properties of Manganese Oxides, edited by C.N.R. Rao and B. Raveau (World Scientific, Singapore, 1998).

${ }^{7}$ S. Yunoki, J. Hu, A. Malvezzi, A. Moreo, N. Furukawa, and E. Dagotto, Phys. Rev. Lett. 80, 845 (1998).

${ }^{8}$ E. Dagotto, S. Yunoki, A.L. Malvezzi, A. Moreo, J. Hu, S. Capponi, D. Poilblanc, and N. Furukawa, Phys. Rev. B 58, 6414 (1998).

${ }^{9}$ S. Yunoki and A. Moreo, Phys. Rev. B 58, 6403 (1998).

${ }^{10}$ D. Arovas and F. Guinea, Phys. Rev. B 58, 9150 (1998).

${ }^{11}$ S. Yunoki, A. Moreo, and E. Dagotto, Phys. Rev. Lett. 81, 5612 (1998).

${ }^{12}$ A. Moreo, S. Yunoki, and E. Dagotto, Science 283, 2034 (1999).

${ }^{13}$ A. Moreo, S. Yunoki, and E. Dagotto, Phys. Rev. Lett. 83, 2773 (1999)

${ }^{14}$ D.S. Dessau, C.-H. Park, T. Saitoh, Z.-X. Shen, P. Villella, N. Hamada, Y. Moritomo, and Y. Tokura, Phys. Rev. Lett. 81, 192 (1998); D.S. Dessau (private communication)

${ }^{15}$ J.M. De Teresa, M.R. Ibarra, P.A. Algarabel, C. Ritter, C. Marquina, J. Blasco, J. García, A. del Moral, and Z. Arnold, Nature (London) 386, 256 (1997); J.B. Goodenough and J.-S. Zhu, ibid. 386, 229 (1997); G. Allodi, R. De Renzi, G. Guidi, F. Lucci, and M.W. Pieper, Phys. Rev. B 56, 6036 (1997); D.E. Cox, P.G. Radaelli, M. Marezio, and S.-W. Cheong, ibid. 57, 3305 (1998); M. Hennion, F. Moussa, G. Biotteau, J. Rodrguez-Carvajal, L. Pinsard, and A. Revcolevschi, Phys. Rev. Lett. 81, 1957 (1998); Wei Bao, C.H. Chen, S.A. Carter, and S.-W. Cheong, Solid State Commun. 98, 55 (1996); M. Roy, J.F. Mitchell, A.P. Ramirez, and P. Schiffer, Phys. Rev. B 58, 5185 (1998); C.H. Booth, F. Bridges, G.H. Kwei, J.M. Lawrence, A.L. Cornelius, and J.J. Neumeier, Phys. Rev. Lett. 80, 853 (1998); Phys. Rev. B 57, 10440 (1998); M. Jaime, P. Lin, S.H. Chun, M.B. Salamon, P. Dorsey, and M. Rubinstein, Phys. Rev. B 60, 1028 (1999); J.-S. Zhou and J.B. Goodenough, Phys. Rev. Lett. 80, 2665 (1998). For more references on inhomogeneities in manganites, see Ref. 12.

${ }^{16}$ M. Fäth, S. Freisem, A. Menovsky, Y. Tomioka, J. Aarts, and J. Mydosh, Science 285, 1540 (1999).

${ }^{17}$ M. Uehara, S. Mori, C.H. Chen, and S.-W. Cheong, Nature (London) 399, 560 (1999).

${ }^{18}$ A. Moreo, M. Mayr, A. Feiguin, S. Yunoki, and E. Dagotto, Phys. Rev. Lett. 84, 5568 (2000).

${ }^{19}$ T. Hotta, Y. Takada, and H. Koizumi, Int. J. Mod. Phys. B 12, 3437 (1998).
${ }^{20}$ T. Hotta, Y. Takada, H. Koizumi, and E. Dagotto, Phys. Rev. Lett. 84, 2477 (2000).

${ }^{21}$ T. Hotta and E. Dagotto, Phys. Rev. B 61, R11 879 (2000).

${ }^{22}$ S. Yunoki, T. Hotta, and E. Dagotto, Phys. Rev. Lett. 84, 3714 (2000).

${ }^{23}$ J. Goodenough, Phys. Rev. 100, 564 (1955).

${ }^{24}$ L.M. Roth, Phys. Rev. 141, 306 (1966); K.I. Kugel and D.I. Khomskii, Usp. Fiz. Nauk. 136, 621 (1981) [Sov. Phys. Usp. 25, 231 (1982)]; S. Inagaki, J. Phys. Soc. Jpn. 39, 596 (1975); M. Cyrot and C. Lyon-Caen, J. Phys. (Paris) 36, 253 (1975); W. Koshibae, Y. Kawamura, S. Ishihara, T. Okamoto, J. Inoue, and S. Maekawa, J. Phys. Soc. Jpn. 66, 957 (1997); L.F. Feiner and A.M. Oleś, Phys. Rev. B 59, 3295 (1999); J. van den Brink, P. Horsch, F. Mack, and A.M. Oleś, ibid. 59, 6795 (1999).

${ }^{25}$ S. Ishihara, J. Inoue, and S. Maekawa, Phys. Rev. B 55, 8280 (1997).

${ }^{26}$ R. Maezono, S. Ishihara, and N. Nagaosa, Phys. Rev. B 57, R13 993 (1998).

${ }^{27}$ T. Mizokawa and A. Fujimori, Phys. Rev. B 56, R493 (1997).

${ }^{28}$ N. Furukawa, J. Phys. Soc. Jpn. 63, 3214 (1994); 63, 2754 (1995).

${ }^{29}$ J.C. Slater and G.F. Koster, Phys. Rev. 94, 1498 (1954).

${ }^{30}$ A.E. Bacquet, T. Mizokawa, T. Saitoh, H. Namatame, and A. Fujimori, Phys. Rev. B 46, 3771 (1992); A. Chainani, M. Mathew, and D.D. Sarma, ibid. 47, 15397 (1993); T. Arima, Y. Tokura, and J.B. Torrance, ibid. 48, 17006 (1993); T. Saitoh, A.E. Bocquet, T. Mizokawa, T. Saitoh, H. Namatame, A. Fujimori, M. Abbate, Y. Takeda, and M. Takano, ibid. 51, 13942 (1995).

${ }^{31}$ G. Perring, G. Aeppli, Y. Moritomo, and Y. Tokura, Phys. Rev. Lett. 78, 3197 (1997).

${ }^{32}$ J. Kanamori, J. Appl. Phys. 31, S14 (1960).

${ }^{33}$ A.J. Millis, P.B. Littlewood, and B.I. Shraiman, Phys. Rev. Lett. 74, 5144 (1995); A.J. Millis, Phys. Rev. B 53, 8434 (1996).

${ }^{34}$ P.B. Allen and Vasili Perebeinos, Phys. Rev. B 60, 10747 (1999).

${ }^{35}$ In general, the adiabaticity is judged from the ratio $\eta=\tau_{\mathrm{e}} / \tau_{\mathrm{i}}$, where $\tau_{\mathrm{e}}$ and $\tau_{\mathrm{i}}$ are characteristic time scales for the motion of electrons and ions, respectively. If $\eta$ is much less than unity, the adiabatic approximation holds, since the motion of ions is very slow compared to that of electrons. Due to the relations $\tau_{\mathrm{i}}$ $\sim \omega^{-1}$ and $\tau_{\mathrm{e}} \sim t^{-1}$, where $\omega$ is the frequency of the lattice distortion, $\eta \sim \omega / t$ is obtained. In manganites, $t$ is $0.2-0.5 \mathrm{eV}$ (Ref. 30), while $\omega$ is about $500-600 \mathrm{~cm}^{-1}$ (Ref. 36). Thus, the adiabatic approximation is, in principle, valid in the study of the JT distortion of manganites.

${ }^{36}$ M.N. Iliev, M.V. Abrashev, V.N. Popov, Y.Y. Sun, C. Thomsen, R.L. Meng, and C.W. Chu, Phys. Rev. B 57, 2872 (1998).

${ }^{37}$ A comment about the cooperative JT effect which is not included in the JT-phononic model is discussed. In principle, a Heisenberg-like coupling between nearest-neighbor phonons should also be added to account for the fact that when a given octahedra is elongated in one direction, its neighbors deform in an opposite way (Ref. 32). However, this cooperative effect has not been studied in detail using computational techniques and here the focus of the paper will be on results obtained with noncooperative phonons. The rich phase diagram and good qualitative agreement with experiments justifies a posteriori this extra approximation. In fact, in the undoped limit, there are no essential differences between the results with and without the cooperative effect (Ref. 38). 
${ }^{38}$ T. Hotta, S. Yunoki, M. Mayr, and E. Dagotto, Phys. Rev. B 60, R15 009 (1999); T. Hotta, S. Yunoki, and E. Dagotto, cond-mat/9907430 (unpublished).

${ }^{39}$ Although the region of small $J_{\mathrm{H}}$ is not realistic to manganites, this region may be interesting from the viewpoint of the competition between the on-site Coulomb repulsion and the attraction mediated by the JT phonons. If $J_{\mathrm{H}}$ is smaller than $E_{\mathrm{JT}}$, there is an energy gain when two electrons with up and down spins occupy the same orbital. It has been suggested that such an effective attraction is important in some semiconductors. See, for instance, G.A. Baraff et al., Phys. Rev. Lett. 43, 956 (1979); Phys. Rev. B 21, 3563 (1980); 21, 5662 (1980).

${ }^{40}$ E. Müller-Hartmann and E. Dagotto, Phys. Rev. B 54, R6819 (1996).

${ }^{41}$ See, for instance, T. Mutou and H. Kontani, Phys. Rev. Lett. 83, 3685 (1999).

${ }^{42}$ If $\beta$ is unrealistically small and $E_{\mathrm{br}}$ becomes much larger than $U^{\prime}, \widetilde{U}^{\prime}$ can be negative, leading to an effective attraction between $e_{\mathrm{g}}$ electrons, and charge-density-wave state or $s$-wave superconducting phase. If the dynamical effect is correctly taken into account beyond the adiabatic approximation, the competition among charge-density-wave, spin-density-wave, and $d$-wave superconducting states will occur, as shown by T. Hotta and Y. Takada, Phys. Rev. B 56, 13916 (1997).

${ }^{43}$ Note that this statement is shown to be valid in the limit of $J_{\mathrm{H}}$ $=\infty$. For finite $J_{\mathrm{H}}$, it is an open problem whether the MFA becomes exact in the $1 \mathrm{D}$ case or not.

${ }^{44}$ For $U^{\prime}=0$ in the one-orbital model, the $1 \mathrm{D}$ system will be insulating if the electron-lattice coupling is nonzero. In the twoorbital case, the situation is not so simple, and the metalinsulator transition point may depend on the hopping matrix. However, at least for the realistic hopping amplitude, the 1D system is insulating for a finite electron-phonon coupling. On the other hand, in higher dimensions, a metal-insulator transition occurs at some finite value of $E_{\mathrm{JT}}$. If $E_{\mathrm{JT}}$ is larger than this critical value, the ground-state wave function will be smoothly changed as $U^{\prime}$ increases. However, when $E_{\mathrm{JT}}$ is so small that the system is metallic at $U^{\prime}=0$, the inclusion of $U^{\prime}$ will bring the metal-insulator transition at some value of $U^{\prime}$. The MFA cannot predict correctly this metal-insulator transition point, but in this paper, the CO state is mainly discussed. Namely, $U^{\prime}\left(E_{\mathrm{JT}}\right)$ is included into the insulating phase originating from $E_{\mathrm{JT}}\left(U^{\prime}\right)$. Although the properties of the metal-insulator transition in manganites are quite interesting, this type of analysis will be left for the future.

${ }^{45}$ If an appropriate mean-field treatment is applied to the full Hamiltonian (1), the orbital set in the OO phase may be determined from the energetical viewpoint even in the purely Coulombic model.

${ }^{46}$ L.M. Falicov and J.C. Kimball, Phys. Rev. Lett. 22, 997 (1969).

${ }^{47}$ H. Koizumi, T. Hotta, and Y. Takada, Phys. Rev. Lett. 80, 4518 (1998); 81, 3803 (1998).

${ }^{48}$ Y. Takada, T. Hotta, and H. Koizumi, Int. J. Mod. Phys. B 13, 3778 (1999).

${ }^{49}$ It may be reasonable to interpret that the JT splitting observed in the photoemission experiment is related to $\widetilde{E}_{\mathrm{JT}}$, not to the bare value $E_{\mathrm{JT}}$. If the purely JT-phononic model is used to investigate the manganite, $E_{\mathrm{JT}}$ appearing in the Hamiltonian may be more able to be interpreted as $\widetilde{E}_{\mathrm{JT}}$. In the present work, however, in order to focus on the competition between the JTphononic and Coulombic interactions, $E_{\mathrm{JT}}$ and $U^{\prime}$ are treated separately. As for the stabilization of the CE-type AFM state with charge-stacked structure, values of $E_{\mathrm{JT}}$ and $U^{\prime}$ are not so important. Rather, $E_{\mathrm{JT}}$ large compared to $t$ is essential.

${ }^{50}$ D.S. Dessau and Z.-X. Shen, in Colossal Magnetoresistive Oxides, edited by Y. Tokura (Gordon and Breach, New York, 2000).

${ }^{51}$ T. Arima and Y. Tokura, J. Phys. Soc. Jpn. 64, 2488 (1995).

${ }^{52}$ I.V. Solovyev and K. Terakura, Phys. Rev. Lett. 83, 2825 (1999).

${ }^{53}$ J. van den Brink, G. Khaliulin, and D. Khomskii, Phys. Rev. Lett. 83, 5118 (1999).

${ }^{54}$ In the Monte Carlo simulation, the grand canonical ensemble has been used and the chemical potential $\mu$ was tuned to obtain the target electron density. In such a calculation, the presence of PS was clearly observed by monitoring the density vs $\mu$. There is a range of densities that cannot be stabilized, no matter how carefully $\mu$ is fine tuned. In other words, the plot $\langle n\rangle$ as a function of $\mu$ presents a discontinuity at some particular value of $\mu$. This effect occurs for the one- and two-orbital models, the latter with JT phonons, and in all dimensions of interest. Further evidence of mixed-phase tendencies can be obtained by monitoring the density as a function of Monte Carlo time. In a stable regime, the density does not change much between Monte Carlo configurations, but in the PS region the fluctuations are very strong.

${ }^{55}$ W. Gill and D.J. Scalapino, Phys. Rev. B 35, 215 (1987); J. Kuei and R.T. Scalettar, ibid., 55, 14968 (1997); J. Hirsch, ibid. 56, 11022 (1997); T. Momoi and K. Kubo, ibid. 58, R567 (1998).

${ }^{56}$ S.R. White, Phys. Rev. Lett. 69, 2863 (1992).

${ }^{57}$ This conclusion is similar to the one found in other Monte Carlo simulations of models for manganites where it was observed that PS appears only after the JT phonons are included in the purely Coulombic model. See Y. Motome and M. Imada, Phys. Rev. B 60, 7921 (1999).

${ }^{58}$ J. Zaanen, J. Phys. Chem. Solids 59, 1769 (1998). See also J. Zaanen and O. Gunnarsson, Phys. Rev. B 40, 7391 (1989); D. Poilblanc and T.M. Rice, ibid. 39, 9749 (1989); H. Schulz, Phys. Rev. Lett. 64, 1445 (1990); M. Kato, K. Machida, H. Nakanishi, and M. Fujita, J. Phys. Soc. Jpn. 59, 1047 (1990); P. Prelovsek and X. Zotos, Phys. Rev. B 47, 5984 (1993); Branko P. Stojković, Z.G. Yu, A.R. Bishop, A.H. Castro Neto, and Niels Grønbech-Jensen, Phys. Rev. Lett. 82, 4679 (1999).

${ }^{59}$ V.J. Emery and S.A. Kivelson, Physica C 209, 597 (1993); S.A. Kivelson and V.J. Emery, Synth. Met. 80, 151 (1996), and references therein.

${ }^{60}$ V. Emery, S. Kivelson, and H. Lin, Phys. Rev. Lett. 64, 475 (1990).

${ }^{61}$ Note that in Ref. 60, PS between an antiferromagnet and a ferromagnet was also discussed, within the framework of the very small $J / t$ limit of the $t-J$ model and assuming a fully saturated FM state. The argument in this reference can be applied directly to the case of the one-orbital model for manganites to justify the presence of phase separation in this context.

${ }^{62}$ It is fair to say that the case of the Hubbard model in the limit of a large dimension $D$ is subtle and deserves special discussion. In principle, at $D=\infty$ there are no indications of phase separation in the one-band Hubbard model [for a review see A. Georges et al., Rev. Mod. Phys. 68, 13 (1996)]. However, it is not clear if this can be taken as a counterexample of phase separation in a Mott insulator, as recently remarked by E.W. Carlson et al., Phys. Rev. B 57, 14704 (1998). In most $D=\infty$ investigations the ratio $J / t$ scales to zero like $1 / \sqrt{D}$ and as a consequence the physics of phase separation is difficult to address. In addition, 
when nonbipartite lattices are used, the antiferromagnetic order is frustrated. Then, more work is needed in large dimensions to clarify the effect of doping on correlated insulators. However, note that in calculations for the one-orbital Kondo model for manganites, phase separation has been clearly observed even at $D=\infty$ (see Ref. 7). This suggests that important qualitative differences may exist between the PS phenomena in models for cuprates and manganites.

${ }^{63}$ S. Mori, C.H. Chen, and S.-W. Cheong, Nature (London) 392, 473 (1998).

${ }^{64}$ E. Dagotto, A. Moreo, F. Ortolani, D. Poilblanc, and J. Riera, Phys. Rev. B 45, 10741 (1992).

${ }^{65}$ A. Moreo, D.J. Scalapino, and E. Dagotto, Phys. Rev. B 43, 11442 (1991).

${ }^{66}$ N. Furukawa and M. Imada, J. Phys. Soc. Jpn. 61, 3331 (1992).

${ }^{67}$ W.O. Puttika, M.U. Luchini, and T.M. Rice, Phys. Rev. Lett. 68, 538 (1992).
${ }^{68}$ C.S. Hellberg and E. Manousakis, Phys. Rev. Lett. 78, 4609 (1997).

${ }^{69}$ S.R. White and D. Scalapino, Phys. Rev. B 61, 6320 (2000).

${ }^{70}$ T. Tohyama, C. Gazza, C.T. Shih, Y.C. Chen, T.K. Lee, S. Maekawa, and E. Dagotto, Phys. Rev. B 59, R11 649 (1999).

${ }^{71}$ G. Martins, R. Eder, and E. Dagotto, Phys. Rev. B 60, R3716 (1999); G. Martins, C. Gazza, J.C. Xavier, A. Feiguin, and E. Dagotto, Phys. Rev. Lett. 84, 5844 (2000).

${ }^{72}$ U. Löw, V.J. Emery, K. Fabricius, and S.A. Kivelson, Phys. Rev. Lett. 72, 1918 (1994).

${ }^{73}$ C.P. Lorenz, D.G. Ravenhall, and C.J. Pethick, Phys. Rev. Lett. 70, 379 (1993), and references therein. The authors thank E. Fradkin for bringing these references to our attention.

${ }^{74}$ E.L. Nagaev, Phys. Status Solidi B 186, 9 (1994); E.L. Nagaev, Phys. Usp. 39, 781 (1996).

${ }^{75}$ L.P. Pryadko, S.A. Kivelson, V.J. Emery, Y.B. Bazaliy, and E.A. Demler, Phys. Rev. B 60, 7541 (1999). 